\title{
The Rotational Spectrum of Dichloromethane-Ne: Internal Dynamics and Cl Quadrupolar Hyperfine Effects
}

Laura B. Favero, ${ }^{\mathrm{a}}$ Assimo Maris, ${ }^{\mathrm{b}}$ Laura Paltrinieri, ${ }^{\mathrm{b}}$ Walther Caminati ${ }^{\mathrm{b}, *}$

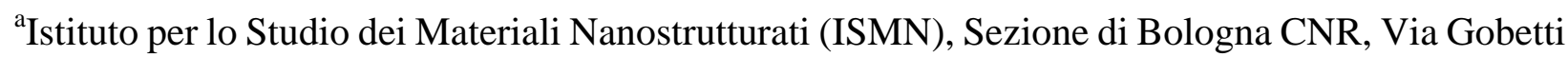
101, I-40129, Bologna, Italy.

bDipartimento di Chimica “G. Ciamician” dell’Università, Via Selmi 2, I-40126 Bologna, Italy.

\section{Supporting Information:}

1) Completion of Ref. [23].

2) Tables with transition frequencies. 


\section{1) Completion of Reference [23].}

M.J. Frisch, G.W. Trucks, H.B. Schlegel, G.E. Scuseria, M.A. Robb, J.R. Cheeseman, G. Scalmani, V. Barone, B. Mennucci, G.A. Petersson, H. Nakatsuji, M. Caricato, X. Li, H.P. Hratchian, A.F. Izmaylov, J. Bloino, G. Zheng, J.L. Sonnenberg, M. Hada, M. Ehara, K. Toyota, R. Fukuda, J. Hasegawa, M. Ishida, T. Nakajima, Y. Honda, O. Kitao, H. Nakai, T. Vreven, J.A. Montgomery, Jr., J.E. Peralta, F. Ogliaro, M. Bearpark, J.J. Heyd, E. Brothers, K.N. Kudin, V.N. Staroverov, T. Keith, R. Kobayashi, J. Normand, K. Raghavachari, A. Rendell, J.C. Burant, S.S. Iyengar, J. Tomasi, M. Cossi, N. Rega, J.M. Millam, M. Klene, J.E. Knox, J. B. Cross, V. Bakken, C. Adamo, J. Jaramillo, R. Gomperts, R.E. Stratmann, O. Yazyev, A.J. Austin, R. Cammi, C.

Pomelli, J.W. Ochterski, R.L. Martin, K. Morokuma, V.G. Zakrzewski, G.A. Voth, P. Salvador, J.J. Dannenberg, S. Dapprich, A.D. Daniels, O. Farkas, J.B. Foresman, J.V. Ortiz, J. Cioslowski, and D.J. Fox, Gaussian 09, Revision D.01, Gaussian, Inc., Wallingford CT, 2013. 
2) Three tables of transition frequencies.

Table 1S. Transition frequencies of DCM-Ne(20) $\mathrm{Cl}(35)-\mathrm{Cl}(35)$.

\begin{tabular}{|c|c|c|c|c|c|c|c|c|c|c|c|c|c|c|c|}
\hline & $J^{\prime}$ & $K_{a}{ }^{\prime}$ & $K_{\mathrm{c}}{ }^{\prime}$ & $v^{\prime}$ & $I^{\prime}$ & $F^{\prime}$ & $J^{\prime \prime}$ & $K_{\mathrm{a}} "$ & $K_{\mathrm{c}}^{\prime}$ & $v^{\prime \prime}$ & $I^{\prime \prime}$ & $F^{\prime \prime}$ & obs & $0-c$ & error \\
\hline & & & & & & & & & & & & & & & \\
\hline \\
\hline 1: & 2 & 1 & 1 & $\odot$ & 1 & 3 & 1 & 0 & 1 & 1 & 3 & 3 & 10377.4420 & 0.0038 & $\odot .002$ \\
\hline 2: & 2 & 1 & 1 & $\odot$ & 2 & $\odot$ & 1 & 0 & 1 & 1 & 0 & 1 & 10379.7846 & $\odot .0 \odot \odot 4$ & 0.002 \\
\hline 3: & 2 & 1 & 1 & $\odot$ & 2 & 1 & 1 & $\odot$ & 1 & 1 & $\odot$ & 1 & 10379.8860 & $\odot .0039$ & $\odot . \odot \odot 2$ \\
\hline 4: & 2 & 1 & 1 & $\odot$ & 3 & 2 & 1 & 0 & 1 & 1 & 3 & 3 & 10380.4032 & 0.0010 & 0.002 \\
\hline 5: & 2 & 1 & 1 & $\odot$ & $\odot$ & 2 & 1 & 0 & 1 & 1 & 2 & 2 & 10380.8727 & $\odot .0049$ & $\odot .0 \odot 2$ \\
\hline 6: & 2 & 1 & 1 & 0 & 1 & 3 & 1 & 0 & 1 & 1 & 1 & 2 & 10382.6125 & 0.0036 & 0.002 \\
\hline 7: & 2 & 1 & 1 & $\odot$ & 1 & 1 & 1 & 0 & 1 & 1 & 1 & $\odot$ & 10385.1324 & 0.0013 & $\odot .002$ \\
\hline 8: & 2 & 1 & 1 & $\odot$ & 3 & 2 & 1 & 0 & 1 & 1 & 1 & 2 & 10385.5738 & $\odot .0008$ & $\odot .002$ \\
\hline 9: & 2 & 1 & 1 & $\odot$ & 1 & 2 & 1 & 0 & 1 & 1 & 1 & 1 & 10387.7855 & 0.0036 & 0.002 \\
\hline 10: & 2 & 1 & 1 & $\odot$ & 3 & 4 & 1 & 0 & 1 & 1 & 3 & 3 & 10389.3261 & 0.0011 & $\odot .0 \odot 2$ \\
\hline 11: & 2 & 1 & 1 & $\odot$ & 3 & 5 & 1 & 0 & 1 & 1 & 3 & 4 & 10389.7943 & 0.0046 & $\odot .002$ \\
\hline 12: & 2 & 1 & 1 & $\odot$ & 2 & 2 & 1 & 0 & 1 & 1 & $\odot$ & 1 & 10390.8984 & -0.0006 & 0.002 \\
\hline 13: & 2 & 1 & 1 & $\odot$ & 3 & 1 & 1 & $\odot$ & 1 & 1 & 3 & 2 & 10391.5452 & 025 & $\odot .0 \odot 2$ \\
\hline 14: & 2 & 1 & 1 & 0 & 2 & 3 & 1 & 0 & 1 & 1 & 2 & 2 & 10391.9181 & 0.0036 & $\odot .0 \odot 2$ \\
\hline 15: & 2 & 1 & 1 & $\odot$ & 2 & 4 & 1 & 0 & 1 & 1 & 2 & 3 & 10391.9560 & $\odot .0044$ & $\odot .002$ \\
\hline 16: & 2 & 1 & 1 & $\odot$ & 3 & 3 & 1 & 0 & 1 & 1 & 3 & 3 & 10392.2665 & $-\odot .0008$ & $\odot .0 \odot 2$ \\
\hline 17: & 2 & 1 & 1 & $\odot$ & $\odot$ & 2 & 1 & 0 & 1 & 1 & 2 & 1 & 10392.9964 & $\odot .0041$ & $\odot .002$ \\
\hline 18: & 2 & 1 & 1 & $\odot$ & 1 & 2 & 1 & 0 & 1 & 1 & 3 & 2 & 10394.1188 & 0.0049 & $\odot .002$ \\
\hline 19: & 2 & 1 & 1 & $\odot$ & 3 & 3 & 1 & 0 & 1 & 1 & 1 & 2 & 10397.4358 & -0.0023 & 0.002 \\
\hline 20: & 2 & 1 & 1 & $\odot$ & 3 & 2 & 1 & 0 & 1 & 1 & 1 & 1 & 10399.5437 & -0.0013 & $\odot .0 \odot 2$ \\
\hline 21: & 2 & 1 & 1 & $\odot$ & 1 & 3 & 1 & 0 & 1 & 1 & 3 & 2 & 10402.9157 & $\odot .0 \odot 27$ & 0.002 \\
\hline 22: & 2 & 1 & 1 & 0 & 2 & 2 & 1 & 0 & 1 & 1 & 2 & 2 & 10403.0105 & -0.0017 & $\odot .0 \odot 2$ \\
\hline 23: & 2 & 1 & 1 & 0 & 2 & 1 & 1 & 0 & 1 & 1 & 2 & 1 & 10404.1188 & $-\odot .0 \odot \odot 9$ & $\odot .0 \odot 2$ \\
\hline 24: & 2 & 1 & 1 & $\odot$ & 3 & 4 & 1 & 0 & 1 & 1 & 3 & 4 & 10404.6 & $-\odot$. & $\odot .0 \odot 2$ \\
\hline 25: & 2 & 1 & 1 & 1 & 3 & 1 & 1 & 0 & 1 & 0 & 1 & 2 & 10384.36 & $-\odot$. & $\odot .0 \odot 2$ \\
\hline 26: & 2 & 1 & 1 & 1 & 1 & 3 & 1 & 0 & 1 & 0 & 3 & 3 & 10390.4998 & $-\odot$. & $\odot .0 \odot 2$ \\
\hline 27: & 2 & 1 & 1 & 1 & 2 & 1 & 1 & 0 & 1 & 0 & 0 & 1 & 10392.9536 & 0. & $\odot .002$ \\
\hline 28: & 2 & 1 & 1 & 1 & 3 & 2 & 1 & 0 & 1 & 0 & 3 & 3 & 10393.4647 & -0. & 0.002 \\
\hline 29: & 2 & 1 & 1 & 1 & $\odot$ & 2 & 1 & 0 & 1 & 0 & 2 & 2 & 10393.9391 & 0.0 & $\odot .0 \odot 2$ \\
\hline 30: & 2 & 1 & 1 & 1 & 1 & 3 & 1 & $\odot$ & 1 & 0 & 1 & 2 & 10395.6801 & $\odot .0 \odot 22$ & $\odot .002$ \\
\hline 31: & 2 & 1 & 1 & 1 & 1 & 1 & 1 & 0 & 1 & 0 & 1 & $\odot$ & $10398.200 \odot$ & $\odot .000 \odot$ & $\odot .0 \odot 2$ \\
\hline 32: & 2 & 1 & 1 & 1 & 3 & 2 & 1 & 0 & 1 & 0 & 1 & 2 & 10398.6377 & -0.0041 & $\odot .0 \odot 2$ \\
\hline 33: & 2 & 1 & 1 & 1 & 1 & 2 & 1 & 0 & 1 & 0 & 1 & 1 & 10400.8519 & 0.0010 & $\odot .002$ \\
\hline $34 /$ & 2 & 1 & 1 & 1 & 3 & 4 & 1 & 0 & 1 & 0 & 3 & 3 & 10402.3863 & $-\odot .0076$ & $\odot .0 \odot 2$ \\
\hline 35: & 2 & 1 & 1 & 1 & 3 & 5 & 1 & 0 & 1 & 0 & 3 & 4 & 10402.8607 & $\odot .0020$ & $\odot .0 \odot 2$ \\
\hline 36: & 2 & 1 & 1 & 1 & 3 & 1 & 1 & 0 & 1 & 0 & 3 & 2 & 10404.6103 & -0.0 & $\odot .0 \odot 2$ \\
\hline 37: & 2 & 1 & 1 & 1 & 2 & 3 & 1 & 0 & 1 & 0 & 2 & 2 & 10404 & 0. & $\odot .002$ \\
\hline 38: & 2 & 1 & 1 & 1 & 2 & 2 & 1 & 0 & 1 & 0 & $\odot$ & 1 & 10403.9684 & $\odot 4$ & $\odot .0 \odot 2$ \\
\hline 39: & 2 & 1 & 1 & 1 & 2 & 4 & 1 & 0 & 1 & 0 & 2 & 3 & 10405.0216 & 0.0 & $\odot .002$ \\
\hline 40: & 2 & 1 & 1 & 1 & 3 & 3 & 1 & 0 & 1 & 0 & 3 & 3 & 10405.3390 & $\odot .0027$ & 0.002 \\
\hline 41: & 2 & 1 & 1 & 1 & $\odot$ & 2 & 1 & 0 & 1 & 0 & 2 & 1 & 10406.0616 & $\odot .0003$ & $\odot .0 \odot 2$ \\
\hline 42: & 2 & 1 & 1 & 1 & 1 & 2 & 1 & 0 & 1 & $\odot$ & 3 & 2 & 10407.1815 & -0.0013 & 0.002 \\
\hline 43: & 2 & 1 & 1 & 1 & 3 & 3 & 1 & 0 & 1 & 0 & 1 & 2 & 10410.5022 & -0.0048 & 0.002 \\
\hline 44: & 2 & 1 & 1 & 1 & 1 & 3 & 1 & 0 & 1 & 0 & 3 & 2 & 10415.9797 & -0.0022 & $\odot .0 \odot 2$ \\
\hline 45: & 2 & 1 & 1 & 1 & 2 & 2 & 1 & 0 & 1 & 0 & 2 & 2 & 10416.0773 & -0.0039 & $\odot .002$ \\
\hline 46: & 2 & 1 & 1 & 1 & 2 & 1 & 1 & 0 & 1 & 0 & 2 & 1 & 10417.1870 & -0.0017 & $\odot .002$ \\
\hline 47: & 2 & 1 & 1 & 1 & 3 & 4 & 1 & 0 & 1 & 0 & 3 & 4 & 10417.7092 & -0.0055 & 0.002 \\
\hline 48: & 2 & 1 & 1 & 1 & 3 & 2 & 1 & 0 & 1 & 0 & 3 & 2 & 10418.9418 & -0.0042 & $\odot .0 \odot 2$ \\
\hline 49: & 2 & 1 & 1 & 1 & 3 & 3 & 1 & 0 & 1 & 0 & 3 & 4 & 10420. & -0 . & $\odot .002$ \\
\hline 50: & 2 & 2 & 1 & $\odot$ & 1 & 1 & 1 & 1 & 1 & 1 & 1 & 1 & 11732 & -0. & 0.002 \\
\hline 51: & 2 & 2 & 1 & $\odot$ & 3 & 3 & 1 & 1 & 1 & 1 & 3 & 4 & 11733.0572 & $-0 . \mathrm{C}-2$. & $\odot .0 \odot 2$ \\
\hline 52: & 2 & 2 & 1 & $\odot$ & 3 & 2 & 1 & 1 & 1 & 1 & 3 & 2 & 11733.2089 & $-0 . \mathrm{C}(x)$ & $\odot .0 \odot 2$ \\
\hline 53: & 2 & 2 & 1 & $\odot$ & 2 & 1 & 1 & 1 & 1 & 1 & 0 & 1 & 11735.2182 & -0.0 & $\odot .0 \odot 2$ \\
\hline 54: & 2 & 2 & 1 & $\odot$ & 3 & 4 & 1 & 1 & 1 & 1 & 3 & 4 & 11735.8102 & -0.0060 & $\odot .0 \odot 2$ \\
\hline 55: & 2 & 2 & 1 & 0 & 1 & 3 & 1 & 1 & 1 & 1 & 3 & 2 & 11735.9358 & -0.0006 & 0.002 \\
\hline 56: & 2 & 2 & 1 & $\odot$ & 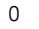 & 2 & 1 & 1 & 1 & 1 & 2 & 2 & 11738.2554 & $-\odot .0003$ & $\odot .002$ \\
\hline 57: & 2 & 2 & 1 & 0 & 3 & 2 & 1 & 1 & 1 & 1 & 1 & 1 & 11740.1262 & -0.0019 & 0.002 \\
\hline 58: & 2 & 2 & 1 & $\odot$ & 1 & 2 & 1 & 1 & 1 & 1 & 3 & 2 & 11744.1495 & -0.0013 & 0.002 \\
\hline 59: & 2 & 2 & 1 & 0 & 3 & 3 & 1 & 1 & 1 & 1 & 1 & 2 & 11744.2178 & -0.0021 & $\odot .0 \odot 2$ \\
\hline 60: & 2 & 2 & 1 & 0 & 2 & 2 & 1 & 1 & 1 & 1 & 0 & 1 & 11745.4239 & $-\odot . \odot \odot \odot 7$ & $\odot .0 \odot 2$ \\
\hline 61: & 2 & 2 & 1 & 0 & 3 & 1 & 1 & 1 & 1 & 1 & 3 & 2 & 11746.5386 & $-\odot .0044$ & $\odot .002$ \\
\hline 62: & 2 & 2 & 1 & 0 & 2 & 4 & 1 & 1 & 1 & 1 & 2 & 3 & 11748.2850 & ๑.0015 & 0.002 \\
\hline 63: & 2 & 2 & 1 & 0 & 2 & 3 & 1 & 1 & 1 & 1 & 2 & 3 & 11748.2850 & -0.0028 & $\odot .0 \odot 2$ \\
\hline 64: & 2 & 2 & 1 & $\odot$ & 2 & 3 & 1 & 1 & 1 & 1 & 2 & 2 & 11748.5118 & 0.0029 & 0.002 \\
\hline 65: & 2 & 2 & 1 & $\odot$ & 3 & 5 & 1 & 1 & 1 & 1 & 3 & 4 & 11749.4863 & -0.0011 & 0.002 \\
\hline 66: & 2 & 2 & 1 & $\odot$ & 3 & 3 & 1 & 1 & 1 & 1 & 3 & 3 & 11749.7350 & -0.0025 & $\odot .0 \odot 2$ \\
\hline 67: & 2 & 2 & 1 & $\odot$ & 1 & 2 & 1 & 1 & 1 & 1 & 1 & 1 & 11751.0656 & 0.0005 & $\odot .002$ \\
\hline 68: & 2 & 2 & 1 & $\odot$ & $\odot$ & 2 & 1 & 1 & 1 & 1 & 2 & 1 & 11751.1412 & $\odot .0006$ & $\odot .002$ \\
\hline 69: & 2 & 2 & 1 & 0 & 3 & 4 & 1 & 1 & 1 & 1 & 3 & 3 & 11752.4888 & -0.0004 & $\odot .002$ \\
\hline 70: & 2 & 2 & 1 & $\odot$ & 3 & 2 & 1 & 1 & 1 & 1 & 1 & 2 & 11755.2047 & $-\odot .00 \odot 1$ & 0.002 \\
\hline 71: & 2 & 2 & 1 & $\odot$ & 1 & 1 & 1 & 1 & 1 & 1 & 1 & 0 & 11756.8913 & $-0.0 \odot 25$ & $\odot .0 \odot 2$ \\
\hline 72: & 2 & 2 & 1 & 0 & 1 & 3 & 1 & 1 & 1 & 1 & 1 & 2 & 11757.9304 & 0.0030 & $\odot .0 \odot 2$ \\
\hline 73: & 2 & 2 & 1 & $\odot$ & 2 & 2 & 1 & 1 & 1 & 1 & 2 & 2 & 1758.7512 & $\odot .0045$ & $\odot .0 \odot 2$ \\
\hline
\end{tabular}




\begin{tabular}{|c|c|c|c|c|c|c|c|c|c|c|c|}
\hline 74: & 2 & 2 & 1 & $\odot$ & 3 & 2 & 1 & 1 & 1 & 1 & 3 \\
\hline 75: & 2 & 2 & 1 & 0 & 2 & 1 & 1 & 1 & 1 & 1 & 2 \\
\hline 76: & 2 & 2 & 1 & 0 & 1 & 3 & 1 & 1 & 1 & 1 & 3 \\
\hline 77: & 2 & 2 & 1 & $\odot$ & 3 & 1 & 1 & 1 & 1 & 1 & 1 \\
\hline 78: & 2 & 2 & 1 & $\odot$ & 1 & 2 & 1 & 1 & 1 & 1 & 3 \\
\hline 79: & 2 & 2 & 1 & 1 & $\odot$ & 2 & 1 & 1 & 1 & $\odot$ & $\odot$ \\
\hline 80: & 2 & 2 & 1 & 1 & 1 & 1 & 1 & 1 & 1 & $\odot$ & 1 \\
\hline 81: & 2 & 2 & 1 & 1 & 3 & 3 & 1 & 1 & 1 & $\odot$ & 3 \\
\hline 82: & 2 & 2 & 1 & 1 & 3 & 2 & 1 & 1 & 1 & $\odot$ & 3 \\
\hline 83: & 2 & 2 & 1 & 1 & 2 & 1 & 1 & 1 & 1 & $\odot$ & $\odot$ \\
\hline 84: & 2 & 2 & 1 & 1 & 3 & 4 & 1 & 1 & 1 & $\odot$ & 3 \\
\hline 85: & 2 & 2 & 1 & 1 & $\odot$ & 2 & 1 & 1 & 1 & $\odot$ & 2 \\
\hline 86: & 2 & 2 & 1 & 1 & 3 & 2 & 1 & 1 & 1 & $\odot$ & 1 \\
\hline 87: & 2 & 2 & 1 & 1 & 1 & 2 & 1 & 1 & 1 & $\odot$ & 3 \\
\hline 88: & 2 & 2 & 1 & 1 & 3 & 3 & 1 & 1 & 1 & $\odot$ & 1 \\
\hline 89: & 2 & 2 & 1 & 1 & 2 & 2 & 1 & 1 & 1 & $\odot$ & $\odot$ \\
\hline 90: & 2 & 2 & 1 & 1 & 3 & 1 & 1 & 1 & 1 & $\odot$ & 3 \\
\hline 91: & 2 & 2 & 1 & 1 & 2 & 4 & 1 & 1 & 1 & $\odot$ & 2 \\
\hline $92 /$ & 2 & 2 & 1 & 1 & 2 & 3 & 1 & 1 & 1 & $\odot$ & 2 \\
\hline 93: & 2 & 2 & 1 & 1 & 2 & 3 & 1 & 1 & 1 & $\odot$ & 2 \\
\hline 94: & 2 & 2 & 1 & 1 & 3 & 5 & 1 & 1 & 1 & $\odot$ & 3 \\
\hline 95: & 2 & 2 & 1 & 1 & 3 & 3 & 1 & 1 & 1 & $\odot$ & 3 \\
\hline 96: & 2 & 2 & 1 & 1 & 1 & 2 & 1 & 1 & 1 & $\odot$ & 1 \\
\hline 97: & 2 & 2 & 1 & 1 & $\odot$ & 2 & 1 & 1 & 1 & $\odot$ & 2 \\
\hline 98: & 2 & 2 & 1 & 1 & 3 & 4 & 1 & 1 & 1 & $\odot$ & 3 \\
\hline 99: & 2 & 2 & 1 & 1 & 3 & 2 & 1 & 1 & 1 & 0 & 1 \\
\hline 100: & 2 & 2 & 1 & 1 & 1 & 1 & 1 & 1 & 1 & $\odot$ & 1 \\
\hline 101: & 2 & 2 & 1 & 1 & 1 & 3 & 1 & 1 & 1 & $\odot$ & 1 \\
\hline 102: & 2 & 2 & 1 & 1 & 2 & 2 & 1 & 1 & 1 & $\odot$ & 2 \\
\hline 103: & 2 & 2 & 1 & 1 & 3 & 2 & 1 & 1 & 1 & $\odot$ & 3 \\
\hline 104: & 2 & 2 & 1 & 1 & 2 & 1 & 1 & 1 & 1 & $\odot$ & 2 \\
\hline 105: & 2 & 2 & 1 & 1 & 1 & 3 & 1 & 1 & 1 & $\odot$ & 3 \\
\hline 106: & 2 & 2 & $\odot$ & 0 & $\odot$ & 2 & 1 & 1 & $\odot$ & 1 & 2 \\
\hline 107: & 2 & 2 & $\odot$ & $\odot$ & $\odot$ & 2 & 1 & 1 & $\odot$ & 1 & $\odot$ \\
\hline 108: & 2 & 2 & $\odot$ & 0 & 1 & 3 & 1 & 1 & $\odot$ & 1 & 1 \\
\hline 109: & 2 & 2 & $\odot$ & 0 & 1 & 2 & 1 & 1 & $\odot$ & 1 & 1 \\
\hline 110: & 2 & 2 & $\odot$ & $\odot$ & 1 & 1 & 1 & 1 & $\odot$ & 1 & 1 \\
\hline 111: & 2 & 2 & $\odot$ & 0 & 1 & 3 & 1 & 1 & $\odot$ & 1 & 3 \\
\hline 112: & 2 & 2 & $\odot$ & $\odot$ & 1 & 2 & 1 & 1 & $\odot$ & 1 & 3 \\
\hline 113: & 2 & 2 & $\odot$ & $\odot$ & 1 & 2 & 1 & 1 & $\odot$ & 1 & 3 \\
\hline 114: & 2 & 2 & $\odot$ & $\odot$ & 1 & 3 & 1 & 1 & $\odot$ & 1 & 3 \\
\hline 115: & 2 & 2 & $\odot$ & $\odot$ & 1 & 3 & 1 & 1 & $\odot$ & 1 & 3 \\
\hline 116: & 2 & 2 & $\odot$ & $\odot$ & 2 & 4 & 1 & 1 & $\odot$ & 1 & 2 \\
\hline 117: & 2 & 2 & $\odot$ & 0 & 2 & 3 & 1 & 1 & $\odot$ & 1 & 2 \\
\hline 118: & 2 & 2 & $\odot$ & $\odot$ & 2 & 2 & 1 & 1 & $\odot$ & 1 & 2 \\
\hline 119: & 2 & 2 & $\odot$ & $\odot$ & 2 & 1 & 1 & 1 & $\odot$ & 1 & $\odot$ \\
\hline 120: & 2 & 2 & $\odot$ & $\odot$ & 2 & 2 & 1 & 1 & $\odot$ & 1 & $\odot$ \\
\hline 121: & 2 & 2 & $\odot$ & $\odot$ & 3 & 5 & 1 & 1 & $\odot$ & 1 & 3 \\
\hline 122: & 2 & 2 & $\odot$ & $\odot$ & 3 & 4 & 1 & 1 & $\odot$ & 1 & 3 \\
\hline 123: & 2 & 2 & $\odot$ & $\odot$ & 3 & 4 & 1 & 1 & $\odot$ & 1 & 3 \\
\hline 124: & 2 & 2 & $\odot$ & $\odot$ & 3 & 3 & 1 & 1 & $\odot$ & 1 & 3 \\
\hline 125: & 2 & 2 & $\odot$ & $\odot$ & 3 & 2 & 1 & 1 & $\odot$ & 1 & 3 \\
\hline 126: & 2 & 2 & $\odot$ & $\odot$ & 3 & 2 & 1 & 1 & $\odot$ & 1 & 3 \\
\hline 127: & 2 & 2 & $\odot$ & $\odot$ & 3 & 3 & 1 & 1 & $\odot$ & 1 & 3 \\
\hline 128: & 2 & 2 & $\odot$ & 0 & 3 & 3 & 1 & 1 & $\odot$ & 1 & 1 \\
\hline 129: & 2 & 2 & $\odot$ & 0 & 3 & 2 & 1 & 1 & $\odot$ & 1 & 1 \\
\hline 130: & 2 & 2 & $\odot$ & $\odot$ & 3 & 2 & 1 & 1 & $\odot$ & 1 & 1 \\
\hline 131: & 2 & 2 & $\odot$ & $\odot$ & 3 & 1 & 1 & 1 & $\odot$ & 1 & 3 \\
\hline 132: & 2 & 2 & $\odot$ & 0 & 3 & 1 & 1 & 1 & $\odot$ & 1 & 1 \\
\hline 133: & 2 & 2 & $\odot$ & 1 & 0 & 2 & 1 & 1 & $\odot$ & $\odot$ & 2 \\
\hline 134: & 2 & 2 & $\odot$ & 1 & 0 & 2 & 1 & 1 & $\odot$ & $\odot$ & $\odot$ \\
\hline 135: & 2 & 2 & $\odot$ & 1 & $\odot$ & 2 & 1 & 1 & $\odot$ & $\odot$ & 2 \\
\hline 136: & 2 & 2 & $\odot$ & 1 & 1 & 3 & 1 & 1 & $\odot$ & 0 & 1 \\
\hline 137: & 2 & 2 & $\odot$ & 1 & 1 & 2 & 1 & 1 & $\odot$ & $\odot$ & 1 \\
\hline 138: & 2 & 2 & $\odot$ & 1 & 1 & 1 & 1 & 1 & $\odot$ & $\odot$ & 1 \\
\hline 139: & 2 & 2 & $\odot$ & 1 & 1 & 3 & 1 & 1 & $\odot$ & $\odot$ & 3 \\
\hline 140: & 2 & 2 & $\odot$ & 1 & 1 & 2 & 1 & 1 & $\odot$ & $\odot$ & 3 \\
\hline 141: & 2 & 2 & $\odot$ & 1 & 1 & 3 & 1 & 1 & $\odot$ & 0 & 3 \\
\hline 142: & 2 & 2 & $\odot$ & 1 & 2 & 4 & 1 & 1 & $\odot$ & 0 & 2 \\
\hline 143: & 2 & 2 & $\odot$ & 1 & 2 & 3 & 1 & 1 & $\odot$ & $\odot$ & 2 \\
\hline 144: & 2 & 2 & $\odot$ & 1 & 2 & 2 & 1 & 1 & $\odot$ & $\odot$ & 2 \\
\hline 145: & 2 & 2 & $\odot$ & 1 & 2 & 2 & 1 & 1 & $\odot$ & $\odot$ & 2 \\
\hline 146: & 2 & 2 & $\odot$ & 1 & 2 & 1 & 1 & 1 & $\odot$ & $\odot$ & 2 \\
\hline 147: & 2 & 2 & $\odot$ & 1 & 2 & 1 & 1 & 1 & $\odot$ & $\odot$ & $\odot$ \\
\hline 148: & 2 & 2 & $\odot$ & 1 & 2 & 2 & 1 & 1 & $\odot$ & $\odot$ & $\odot$ \\
\hline 149: & 2 & 2 & $\odot$ & 1 & 2 & 2 & 1 & 1 & $\odot$ & $\odot$ & 2 \\
\hline 150: & 2 & 2 & $\odot$ & 1 & 3 & 5 & 1 & 1 & $\odot$ & $\odot$ & 3 \\
\hline 151: & 2 & 2 & $\odot$ & 1 & 3 & 4 & 1 & 1 & $\odot$ & $\odot$ & 3 \\
\hline 152: & 2 & 2 & $\odot$ & 1 & 3 & 4 & 1 & 1 & $\odot$ & $\odot$ & 3 \\
\hline 153: & 2 & 2 & $\odot$ & 1 & 3 & 3 & 1 & 1 & $\odot$ & $\odot$ & 3 \\
\hline 154: & 2 & 2 & $\odot$ & 1 & 3 & 2 & 1 & 1 & $\odot$ & $\odot$ & 3 \\
\hline 155： & 2 & 2 & $\odot$ & 1 & 3 & 3 & 1 & 1 & $\odot$ & $\odot$ & 3 \\
\hline 156: & 2 & 2 & $\odot$ & 1 & 3 & 3 & 1 & 1 & $\odot$ & $\odot$ & 1 \\
\hline 157： & 2 & 2 & $\odot$ & 1 & 3 & 2 & 1 & 1 & $\odot$ & $\odot$ & 1 \\
\hline 158: & 2 & 2 & $\odot$ & 1 & 3 & 2 & 1 & 1 & $\odot$ & $\odot$ & 1 \\
\hline 159: & 2 & 2 & $\odot$ & 1 & 3 & 1 & 1 & 1 & $\odot$ & $\odot$ & 3 \\
\hline 160: & 2 & 2 & $\odot$ & 1 & 3 & 1 & 1 & 1 & $\odot$ & $\odot$ & 1 \\
\hline
\end{tabular}




\begin{tabular}{|c|c|c|c|c|c|c|c|c|c|c|c|}
\hline 161: & 3 & 2 & 1 & $\odot$ & 3 & 2 & 2 & 1 & 1 & 1 & 3 \\
\hline 162: & 3 & 2 & 1 & 0 & 2 & 2 & 2 & 1 & 1 & 1 & 2 \\
\hline 163: & 3 & 2 & 1 & $\odot$ & 1 & 4 & 2 & 1 & 1 & 1 & 3 \\
\hline 164: & 3 & 2 & 1 & 0 & 3 & 1 & 2 & 1 & 1 & 1 & 3 \\
\hline 165: & 3 & 2 & 1 & $\odot$ & $\odot$ & 3 & 2 & 1 & 1 & 1 & 2 \\
\hline 166: & 3 & 2 & 1 & $\odot$ & 3 & 3 & 2 & 1 & 1 & 1 & 3 \\
\hline 167: & 3 & 2 & 1 & $\odot$ & 1 & 3 & 2 & 1 & 1 & 1 & 1 \\
\hline 168: & 3 & 2 & 1 & 0 & 3 & 2 & 2 & 1 & 1 & 1 & 3 \\
\hline 169: & 3 & 2 & 1 & $\odot$ & 3 & 5 & 2 & 1 & 1 & 1 & 3 \\
\hline 170: & 3 & 2 & 1 & $\odot$ & 1 & 2 & 2 & 1 & 1 & 1 & 1 \\
\hline 171: & 3 & 2 & 1 & 0 & 2 & 3 & 2 & 1 & 1 & 1 & 2 \\
\hline 172: & 3 & 2 & 1 & $\odot$ & 3 & 4 & 2 & 1 & 1 & 1 & 3 \\
\hline 173: & 3 & 2 & 1 & $\odot$ & 2 & 5 & 2 & 1 & 1 & 1 & 2 \\
\hline 174: & 3 & 2 & 1 & $\odot$ & 3 & 6 & 2 & 1 & 1 & 1 & 3 \\
\hline 175: & 3 & 2 & 1 & $\odot$ & 3 & 0 & 2 & 1 & 1 & 1 & 3 \\
\hline 176: & 3 & 2 & 1 & $\odot$ & 3 & 4 & 2 & 1 & 1 & 1 & 3 \\
\hline 177: & 3 & 2 & 1 & 0 & 1 & 4 & 2 & 1 & 1 & 1 & 1 \\
\hline 178: & 3 & 2 & 1 & $\odot$ & $\odot$ & 3 & 2 & 1 & 1 & 1 & $\odot$ \\
\hline 179: & 3 & 2 & 1 & $\odot$ & 1 & 3 & 2 & 1 & 1 & 1 & 1 \\
\hline 180: & 3 & 2 & 1 & 0 & 3 & 3 & 2 & 1 & 1 & 1 & 3 \\
\hline 181: & 3 & 2 & 1 & $\odot$ & 3 & 1 & 2 & 1 & 1 & 1 & 3 \\
\hline 182: & 3 & 2 & 1 & $\odot$ & 3 & 2 & 2 & 1 & 1 & 1 & 1 \\
\hline 183: & 3 & 2 & 1 & $\odot$ & 3 & 2 & 2 & 1 & 1 & 1 & 3 \\
\hline 184: & 3 & 2 & 1 & $\odot$ & 3 & 5 & 2 & 1 & 1 & 1 & 3 \\
\hline 185: & 3 & 2 & 1 & 1 & 2 & 2 & 2 & 1 & 1 & $\odot$ & 2 \\
\hline 186: & 3 & 2 & 1 & 1 & $\odot$ & 3 & 2 & 1 & 1 & $\odot$ & 2 \\
\hline 187: & 3 & 2 & 1 & 1 & 3 & 3 & 2 & 1 & 1 & $\odot$ & 3 \\
\hline 188: & 3 & 2 & 1 & 1 & 1 & 3 & 2 & 1 & 1 & $\odot$ & 1 \\
\hline 189: & 3 & 2 & 1 & 1 & 3 & 2 & 2 & 1 & 1 & $\odot$ & 3 \\
\hline 190: & 3 & 2 & 1 & 1 & 3 & 5 & 2 & 1 & 1 & 0 & 3 \\
\hline 191: & 3 & 2 & 1 & 1 & 1 & 2 & 2 & 1 & 1 & $\odot$ & 1 \\
\hline 192: & 3 & 2 & 1 & 1 & 2 & 3 & 2 & 1 & 1 & $\odot$ & 2 \\
\hline 193: & 3 & 2 & 1 & 1 & 3 & 4 & 2 & 1 & 1 & $\odot$ & 3 \\
\hline 194: & 3 & 2 & 1 & 1 & 3 & 6 & 2 & 1 & 1 & $\odot$ & 3 \\
\hline 195: & 3 & 2 & 1 & 1 & 3 & 4 & 2 & 1 & 1 & $\odot$ & 3 \\
\hline 196: & 3 & 2 & 1 & 1 & $\odot$ & 3 & 2 & 1 & 1 & $\odot$ & $\odot$ \\
\hline 197: & 3 & 2 & 1 & 1 & 1 & 4 & 2 & 1 & 1 & $\odot$ & 1 \\
\hline 198: & 3 & 2 & 1 & 1 & 1 & 3 & 2 & 1 & 1 & $\odot$ & 1 \\
\hline 199: & 3 & 2 & 1 & 1 & 3 & 3 & 2 & 1 & 1 & $\odot$ & 3 \\
\hline 200: & 3 & 2 & 1 & 1 & 3 & 1 & 2 & 1 & 1 & $\odot$ & 3 \\
\hline 201: & 3 & 2 & 1 & 1 & 2 & 3 & 2 & 1 & 1 & $\odot$ & 2 \\
\hline 202: & 3 & 2 & 1 & 1 & 3 & 2 & 2 & 1 & 1 & $\odot$ & 3 \\
\hline 203: & 3 & 2 & 1 & 1 & 3 & 5 & 2 & 1 & 1 & $\odot$ & 3 \\
\hline 204: & 3 & 1 & 2 & $\odot$ & 1 & 3 & 2 & 0 & 2 & 1 & 1 \\
\hline 205: & 3 & 1 & 2 & $\odot$ & 3 & 1 & 2 & 0 & 2 & 1 & 3 \\
\hline 206: & 3 & 1 & 2 & $\odot$ & 3 & 2 & 2 & $\odot$ & 2 & 1 & 3 \\
\hline 207: & 3 & 1 & 2 & $\odot$ & $\odot$ & 3 & 2 & 0 & 2 & 1 & 2 \\
\hline 208: & 3 & 1 & 2 & $\odot$ & 1 & 3 & 2 & 0 & 2 & 1 & 1 \\
\hline 209: & 3 & 1 & 2 & $\odot$ & 1 & 4 & 2 & 0 & 2 & 1 & 3 \\
\hline 210: & 3 & 1 & 2 & $\odot$ & 2 & 2 & 2 & 0 & 2 & 1 & $\odot$ \\
\hline 211: & 3 & 1 & 2 & $\odot$ & 3 & 6 & 2 & 0 & 2 & 1 & 3 \\
\hline 212: & 3 & 1 & 2 & $\odot$ & 3 & 2 & 2 & 0 & 2 & 1 & 1 \\
\hline 213: & 3 & 1 & 2 & $\odot$ & 1 & 4 & 2 & $\odot$ & 2 & 1 & 1 \\
\hline 214: & 3 & 1 & 2 & $\odot$ & 2 & 4 & 2 & 0 & 2 & 1 & 2 \\
\hline 215: & 3 & 1 & 2 & $\odot$ & 2 & 5 & 2 & 0 & 2 & 1 & 2 \\
\hline 216: & 3 & 1 & 2 & $\odot$ & 3 & 3 & 2 & 0 & 2 & 1 & 3 \\
\hline 217: & 3 & 1 & 2 & $\odot$ & 3 & 5 & 2 & 0 & 2 & 1 & 3 \\
\hline 218: & 3 & 1 & 2 & $\odot$ & 1 & 2 & 2 & 0 & 2 & 1 & 1 \\
\hline 219: & 3 & 1 & 2 & $\odot$ & 2 & 2 & 2 & 0 & 2 & 1 & 2 \\
\hline 220: & 3 & 1 & 2 & $\odot$ & 3 & 3 & 2 & 0 & 2 & 1 & 3 \\
\hline 221: & 3 & 1 & 2 & $\odot$ & 2 & 3 & 2 & $\odot$ & 2 & 1 & $\odot$ \\
\hline 222: & 3 & 1 & 2 & $\odot$ & 3 & 4 & 2 & 0 & 2 & 1 & 3 \\
\hline 223: & 3 & 1 & 2 & $\odot$ & 3 & 5 & 2 & 0 & 2 & 1 & 3 \\
\hline 224: & 3 & 1 & 2 & $\odot$ & 2 & 3 & 2 & $\odot$ & 2 & 1 & 2 \\
\hline 225: & 3 & 1 & 2 & 1 & 3 & $\odot$ & 2 & $\odot$ & 2 & $\odot$ & 3 \\
\hline 226: & 3 & 1 & 2 & 1 & 1 & 3 & 2 & 0 & 2 & $\odot$ & 1 \\
\hline 227: & 3 & 1 & 2 & 1 & 3 & 1 & 2 & 0 & 2 & $\odot$ & 3 \\
\hline 228: & 3 & 1 & 2 & 1 & 3 & 2 & 2 & 0 & 2 & 0 & 3 \\
\hline 229: & 3 & 1 & 2 & 1 & $\odot$ & 3 & 2 & 0 & 2 & 0 & 2 \\
\hline 230: & 3 & 1 & 2 & 1 & 1 & 3 & 2 & 0 & 2 & $\odot$ & 1 \\
\hline 231: & 3 & 1 & 2 & 1 & 1 & 4 & 2 & 0 & 2 & $\odot$ & 3 \\
\hline 232: & 3 & 1 & 2 & 1 & 3 & 6 & 2 & 0 & 2 & $\odot$ & 3 \\
\hline 233: & 3 & 1 & 2 & 1 & 1 & 4 & 2 & $\odot$ & 2 & $\odot$ & 1 \\
\hline 234: & 3 & 1 & 2 & 1 & 2 & 4 & 2 & 0 & 2 & $\odot$ & 2 \\
\hline 235: & 3 & 1 & 2 & 1 & 2 & 5 & 2 & 0 & 2 & $\odot$ & 2 \\
\hline 236: & 3 & 1 & 2 & 1 & 3 & 3 & 2 & $\odot$ & 2 & 0 & 3 \\
\hline 237: & 3 & 1 & 2 & 1 & 3 & 2 & 2 & 0 & 2 & 0 & 3 \\
\hline 238: & 3 & 1 & 2 & 1 & 3 & 5 & 2 & 0 & 2 & 0 & 3 \\
\hline 239: & 3 & 1 & 2 & 1 & 1 & 2 & 2 & 0 & 2 & $\odot$ & 1 \\
\hline 240: & 3 & 1 & 2 & 1 & 3 & 3 & 2 & 0 & 2 & $\odot$ & 3 \\
\hline 241: & 3 & 1 & 2 & 1 & 2 & 3 & 2 & 0 & 2 & 0 & $\odot$ \\
\hline 242: & 3 & 1 & 2 & 1 & 3 & 4 & 2 & $\odot$ & 2 & $\odot$ & 3 \\
\hline $243 /$ & 3 & 1 & 2 & 1 & 3 & 4 & 2 & 0 & 2 & $\odot$ & 3 \\
\hline 244: & 3 & 1 & 2 & 1 & 3 & 5 & 2 & 0 & 2 & $\odot$ & 3 \\
\hline 245: & 3 & 2 & 2 & $\odot$ & $\odot$ & 3 & 2 & 1 & 2 & 1 & $\odot$ \\
\hline 246: & 3 & 2 & 2 & $\odot$ & $\odot$ & 3 & 2 & 1 & 2 & 1 & 2 \\
\hline 247: & 3 & 2 & 2 & 0 & 1 & 4 & 2 & 1 & 2 & 1 & 1 \\
\hline
\end{tabular}




\begin{tabular}{|c|c|c|c|c|c|c|c|c|c|c|c|}
\hline 248: & 3 & 2 & 2 & 0 & 3 & 3 & 2 & 1 & 2 & 1 & 1 \\
\hline 249: & 3 & 2 & 2 & 0 & 1 & 2 & 2 & 1 & 2 & 1 & 1 \\
\hline 50 : & 3 & 2 & 2 & $\odot$ & 1 & 4 & 2 & 1 & 2 & 1 & 3 \\
\hline 251: & 3 & 2 & 2 & $\odot$ & 1 & 3 & 2 & 1 & 2 & 1 & 3 \\
\hline 252: & 3 & 2 & 2 & $\odot$ & 2 & 5 & 2 & 1 & 2 & 1 & 2 \\
\hline 253: & 3 & 2 & 2 & $\odot$ & 2 & 4 & 2 & 1 & 2 & 1 & 2 \\
\hline $254:$ & 3 & 2 & 2 & 0 & 2 & 3 & 2 & 1 & 2 & 1 & 0 \\
\hline 255: & 3 & 2 & 2 & $\odot$ & 3 & 6 & 2 & 1 & 2 & 1 & 3 \\
\hline 256: & 3 & 2 & 2 & 0 & 3 & 5 & 2 & 1 & 2 & 1 & 3 \\
\hline 257: & 3 & 2 & 2 & $\odot$ & 3 & 4 & 2 & 1 & 2 & 1 & 3 \\
\hline 58: & 3 & 2 & 2 & $\odot$ & 3 & 1 & 2 & 1 & 2 & 1 & 3 \\
\hline 259: & 3 & 2 & 2 & $\odot$ & 3 & 2 & 2 & 1 & 2 & 1 & 3 \\
\hline 260 : & 3 & 2 & 2 & 1 & $\odot$ & 3 & 2 & 1 & 2 & $\odot$ & 0 \\
\hline 261: & 3 & 2 & 2 & 1 & $\odot$ & 3 & 2 & 1 & 2 & $\odot$ & 2 \\
\hline 262: & 3 & 2 & 2 & 1 & 1 & 4 & 2 & 1 & 2 & $\odot$ & 1 \\
\hline $263 /$ & 3 & 2 & 2 & 1 & 1 & 3 & 2 & 1 & 2 & $\odot$ & 1 \\
\hline 264: & 3 & 2 & 2 & 1 & 1 & 2 & 2 & 1 & 2 & $\odot$ & 1 \\
\hline 265: & 3 & 2 & 2 & 1 & 1 & 4 & 2 & 1 & 2 & $\odot$ & 3 \\
\hline 266: & 3 & 2 & 2 & 1 & 1 & 3 & 2 & 1 & 2 & $\odot$ & 3 \\
\hline 267: & 3 & 2 & 2 & 1 & 2 & 5 & 2 & 1 & 2 & $\odot$ & 2 \\
\hline 268: & 3 & 2 & 2 & 1 & 2 & 3 & 2 & 1 & 2 & $\odot$ & 2 \\
\hline 269: & 3 & 2 & 2 & 1 & 2 & 4 & 2 & 1 & 2 & 0 & 2 \\
\hline $270:$ & 3 & 2 & 2 & 1 & 3 & 6 & 2 & 1 & 2 & $\odot$ & 3 \\
\hline 271: & 3 & 2 & 2 & 1 & 3 & 5 & 2 & 1 & 2 & $\odot$ & 3 \\
\hline 272: & 3 & 2 & 2 & 1 & 3 & 1 & 2 & 1 & 2 & 0 & 3 \\
\hline 273: & 3 & 3 & 1 & $\odot$ & 3 & 3 & 2 & 2 & 1 & 1 & 3 \\
\hline 274: & 3 & 3 & 1 & $\odot$ & 3 & 4 & 2 & 2 & 1 & 1 & 3 \\
\hline 275: & 3 & 3 & 1 & $\odot$ & 3 & 4 & 2 & 2 & 1 & 1 & 3 \\
\hline 276: & 3 & 3 & 1 & $\odot$ & 1 & 4 & 2 & 2 & 1 & 1 & 1 \\
\hline 277: & 3 & 3 & 1 & $\odot$ & $\odot$ & 3 & 2 & 2 & 1 & 1 & $\odot$ \\
\hline $278 /$ & 3 & 3 & 1 & $\odot$ & 3 & 1 & 2 & 2 & 1 & 1 & 3 \\
\hline 279: & 3 & 3 & 1 & $\odot$ & 2 & 5 & 2 & 2 & 1 & 1 & 2 \\
\hline 280: & 3 & 3 & 1 & $\odot$ & 1 & 3 & 2 & 2 & 1 & 1 & 1 \\
\hline 281: & 3 & 3 & 1 & $\odot$ & 2 & 3 & 2 & 2 & 1 & 1 & 2 \\
\hline 282: & 3 & 3 & 1 & 0 & 3 & 5 & 2 & 2 & 1 & 1 & 3 \\
\hline 283: & 3 & 3 & 1 & 0 & 3 & 6 & 2 & 2 & 1 & 1 & 3 \\
\hline 284: & 3 & 3 & 1 & 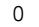 & 3 & 3 & 2 & 2 & 1 & 1 & 3 \\
\hline 285: & 3 & 3 & 1 & 0 & 3 & 2 & 2 & 2 & 1 & 1 & 3 \\
\hline 286: & 3 & 3 & 1 & $\odot$ & 2 & 2 & 2 & 2 & 1 & 1 & $\odot$ \\
\hline 287: & 3 & 3 & 1 & $\odot$ & 2 & 3 & 2 & 2 & 1 & 1 & 2 \\
\hline 288: & 3 & 3 & 1 & 1 & 3 & 5 & 2 & 2 & 1 & 0 & 3 \\
\hline 289: & 3 & 3 & 1 & 1 & 3 & 2 & 2 & 2 & 1 & $\odot$ & 3 \\
\hline 290: & 3 & 3 & 1 & 1 & 3 & 3 & 2 & 2 & 1 & 0 & 3 \\
\hline 291: & 3 & 3 & 1 & 1 & 3 & 4 & 2 & 2 & 1 & $\odot$ & 3 \\
\hline 292: & 3 & 3 & 1 & 1 & 3 & 4 & 2 & 2 & 1 & $\odot$ & 3 \\
\hline 293: & 3 & 3 & 1 & 1 & 1 & 4 & 2 & 2 & 1 & $\odot$ & 1 \\
\hline $294:$ & 3 & 3 & 1 & 1 & $\odot$ & 3 & 2 & 2 & 1 & 0 & $\odot$ \\
\hline 295: & 3 & 3 & 1 & 1 & 3 & 1 & 2 & 2 & 1 & 0 & 3 \\
\hline 296: & 3 & 3 & 1 & 1 & 2 & 5 & 2 & 2 & 1 & 0 & 2 \\
\hline 297: & 3 & 3 & 1 & 1 & 1 & 3 & 2 & 2 & 1 & 0 & 1 \\
\hline 298: & 3 & 3 & 1 & 1 & 1 & 2 & 2 & 2 & 1 & 0 & 1 \\
\hline 299: & 3 & 3 & 1 & 1 & 2 & 3 & 2 & 2 & 1 & 0 & 2 \\
\hline $300:$ & 3 & 3 & 1 & 1 & 3 & 5 & 2 & 2 & 1 & 0 & 3 \\
\hline 301: & 3 & 3 & 1 & 1 & 3 & 6 & 2 & 2 & 1 & $\odot$ & 3 \\
\hline 302: & 3 & 3 & 1 & 1 & 3 & 0 & 2 & 2 & 1 & 0 & 3 \\
\hline 303: & 3 & 3 & 1 & 1 & 3 & 3 & 2 & 2 & 1 & 0 & 3 \\
\hline $304:$ & 3 & 3 & 1 & 1 & 3 & 2 & 2 & 2 & 1 & 0 & 3 \\
\hline 305: & 3 & 3 & 1 & 1 & 1 & 3 & 2 & 2 & 1 & $\odot$ & 1 \\
\hline 306: & 3 & 3 & 0 & $\odot$ & 2 & 3 & 2 & 2 & $\odot$ & 1 & 2 \\
\hline 307: & 3 & 3 & $\odot$ & 0 & 3 & 5 & 2 & 2 & $\odot$ & 1 & 3 \\
\hline 308: & 3 & 3 & 0 & 0 & 2 & 3 & 2 & 2 & $\odot$ & 1 & 0 \\
\hline 309: & 3 & 3 & $\odot$ & $\odot$ & 3 & 4 & 2 & 2 & $\odot$ & 1 & 3 \\
\hline 310: & 3 & 3 & $\odot$ & 0 & 3 & 3 & 2 & 2 & $\odot$ & 1 & 3 \\
\hline 311: & 3 & 3 & $\odot$ & $\odot$ & 1 & 2 & 2 & 2 & $\odot$ & 1 & 1 \\
\hline 312: & 3 & 3 & $\odot$ & $\odot$ & 3 & 5 & 2 & 2 & $\odot$ & 1 & 3 \\
\hline 313: & 3 & 3 & $\odot$ & 0 & 2 & 5 & 2 & 2 & 0 & 1 & 2 \\
\hline 314: & 3 & 3 & $\odot$ & $\odot$ & 2 & 4 & 2 & 2 & $\odot$ & 1 & 2 \\
\hline 315: & 3 & 3 & $\odot$ & 0 & 1 & 4 & 2 & 2 & 0 & 1 & 1 \\
\hline 316: & 3 & 3 & $\odot$ & $\odot$ & 3 & 6 & 2 & 2 & $\odot$ & 1 & 3 \\
\hline 317: & 3 & 3 & 0 & 0 & 1 & 3 & 2 & 2 & $\odot$ & 1 & 1 \\
\hline 318: & 3 & 3 & $\odot$ & 0 & $\odot$ & 3 & 2 & 2 & $\odot$ & 1 & 2 \\
\hline 319: & 3 & 3 & 0 & 0 & $\odot$ & 3 & 2 & 2 & $\odot$ & 1 & 2 \\
\hline 320: & 3 & 3 & 0 & 1 & 3 & 5 & 2 & 2 & $\odot$ & $\odot$ & 3 \\
\hline 321: & 3 & 3 & $\odot$ & 1 & 3 & 4 & 2 & 2 & $\odot$ & $\odot$ & 3 \\
\hline 322: & 3 & 3 & $\odot$ & 1 & 3 & 4 & 2 & 2 & $\odot$ & 0 & 3 \\
\hline 323: & 3 & 3 & $\odot$ & 1 & 2 & 3 & 2 & 2 & $\odot$ & 0 & 0 \\
\hline 324: & 3 & 3 & $\odot$ & 1 & 3 & 3 & 2 & 2 & $\odot$ & $\odot$ & 3 \\
\hline 325: & 3 & 3 & $\odot$ & 1 & 1 & 2 & 2 & 2 & $\odot$ & $\odot$ & 1 \\
\hline 326: & 3 & 3 & $\odot$ & 1 & 3 & 5 & 2 & 2 & $\odot$ & $\odot$ & 3 \\
\hline 327: & 3 & 3 & $\odot$ & 1 & 3 & 2 & 2 & 2 & $\odot$ & 0 & 3 \\
\hline 328: & 3 & 3 & $\odot$ & 1 & 2 & 5 & 2 & 2 & $\odot$ & 0 & 2 \\
\hline 329: & 3 & 3 & $\odot$ & 1 & 2 & 4 & 2 & 2 & $\odot$ & $\odot$ & 2 \\
\hline 330: & 3 & 3 & $\odot$ & 1 & 1 & 4 & 2 & 2 & $\odot$ & $\odot$ & 1 \\
\hline 331: & 3 & 3 & 0 & 1 & 3 & 3 & 2 & 2 & $\odot$ & $\odot$ & 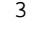 \\
\hline 332: & 3 & 3 & $\odot$ & 1 & 3 & 6 & 2 & 2 & $\odot$ & $\odot$ & 3 \\
\hline 333: & 3 & 3 & $\odot$ & 1 & 1 & 3 & 2 & 2 & $\Theta$ & 0 & 1 \\
\hline $334:$ & 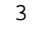 & 3 & $\odot$ & 1 & 0 & 3 & 2 & 2 & $\odot$ & $\odot$ & 2 \\
\hline
\end{tabular}


$\begin{array}{llllllllllllllll}335: & 3 & 3 & \odot & 1 & 3 & 1 & 2 & 2 & \odot & \odot & 3 & 1 & 17458.1614 & -0.0039 & 0.002\end{array}$

$\begin{array}{llllllllllllllll}336: & 3 & 3 & 0 & 1 & 1 & 4 & 2 & 2 & 0 & 0 & 3 & 4 & 17458.2725 & -0.0061 & 0.002\end{array}$

$\begin{array}{llllllllllllllll}337: & 3 & 3 & \odot & 1 & 3 & 2 & 2 & 2 & \odot & 0 & 3 & 2 & 17458.6830 & -0.0050 & 0.002\end{array}$

$\begin{array}{llllllllllllllll}338: & 3 & 3 & \odot & 1 & 1 & 3 & 2 & 2 & \odot & 0 & 1 & 3 & 17461.3704 & -0.0054 & 0.002\end{array}$ 
Table 2S. Transition frequencies of DCM-Ne(22) Cl(35)-Cl(35)

\begin{tabular}{|c|c|c|c|c|c|c|c|c|c|c|c|c|c|c|c|}
\hline & $J^{\prime}$ & $K_{\mathrm{a}}{ }^{\prime}$ & $K_{\mathrm{c}}{ }^{\prime}$ & $v^{\prime}$ & $I^{\prime}$ & $F^{\prime}$ & $J^{\prime \prime}$ & $K_{\mathrm{a}} "$ & $K_{\mathrm{c}}{ }^{\prime \prime}$ & $v^{\prime \prime}$ & $I^{\prime \prime}$ & $F^{\prime}$ & obs & $0-c$ & error \\
\hline 1: & 2 & 1 & 1 & $\odot$ & 2 & 1 & 1 & $\odot$ & 1 & 1 & $\odot$ & 1 & $9896.0 \odot 82$ & 0.0036 & 0.002 \\
\hline 2: & 2 & 1 & 1 & $\odot$ & $\odot$ & 2 & 1 & 0 & 1 & 1 & 2 & 2 & 9896.9852 & 0.0019 & 0.002 \\
\hline 3: & 2 & 1 & 1 & $\odot$ & 1 & 3 & 1 & $\odot$ & 1 & 1 & 1 & 2 & 9898.7227 & $\odot .0 \odot 23$ & $\odot . \odot \odot 2$ \\
\hline 4: & 2 & 1 & 1 & $\odot$ & 3 & 2 & 1 & 0 & 1 & 1 & 1 & 2 & 9901.6847 & $\odot . \odot \odot \odot \odot$ & $\odot . \odot \odot 2$ \\
\hline 5: & 2 & 1 & 1 & $\odot$ & 1 & 2 & 1 & 0 & 1 & 1 & 1 & 1 & 9903.8998 & 0.0012 & 0.002 \\
\hline 6: & 2 & 1 & 1 & $\odot$ & 3 & 4 & 1 & 0 & 1 & 1 & 3 & 3 & 9905.4401 & $\odot .0009$ & 0.002 \\
\hline 7: & 2 & 1 & 1 & $\odot$ & 3 & 5 & 1 & 0 & 1 & 1 & 3 & 4 & 9905.9012 & $\odot .0 \odot 25$ & $\odot . \odot \odot 2$ \\
\hline 8: & 2 & 1 & 1 & $\odot$ & 2 & 2 & 1 & 0 & 1 & 1 & $\odot$ & 1 & 9907.0118 & $-\odot .0006$ & $\odot .002$ \\
\hline 9: & 2 & 1 & 1 & $\odot$ & 3 & 1 & 1 & $\odot$ & 1 & 1 & 3 & 2 & 9907.6594 & $\odot .0009$ & $\odot .0 \odot 2$ \\
\hline 10: & 2 & 1 & 1 & $\odot$ & 2 & 4 & 1 & $\odot$ & 1 & 1 & 2 & 3 & 9908 & $\odot .0010$ & $\odot .0 \odot 2$ \\
\hline 11: & 2 & 1 & 1 & 0 & 3 & 3 & 1 & 0 & 1 & 1 & 3 & 3 & 990 & -0 & .002 \\
\hline 12: & 2 & 1 & 1 & $\odot$ & 0 & 2 & 1 & $\odot$ & 1 & 1 & 2 & 1 & 990 & 22 & $.0 \odot 2$ \\
\hline 13: & 2 & 1 & 1 & $\odot$ & 1 & 2 & 1 & $\odot$ & 1 & 1 & 3 & 2 & 9910.2287 & $\odot .0001$ & $\odot . \odot \odot 2$ \\
\hline 14: & 2 & 1 & 1 & $\odot$ & 3 & 3 & 1 & $\odot$ & 1 & 1 & 1 & 2 & 9913.5459 & $-\odot .0 \odot 22$ & ๑. 002 \\
\hline 15: & 2 & 1 & 1 & $\odot$ & 1 & 3 & 1 & 0 & 1 & 1 & 3 & 2 & 9919.0161 & $-\odot .0 \odot 24$ & $\odot .0 \odot 2$ \\
\hline 16: & 2 & 1 & 1 & 1 & 3 & 1 & 1 & $\odot$ & 1 & 0 & 1 & 2 & 9899.5296 & $-\odot . \odot \odot 27$ & $\odot .002$ \\
\hline 17: & 2 & 1 & 1 & 1 & 1 & 3 & 1 & 0 & 1 & 0 & 3 & 3 & 9905.7293 & $\odot .0064$ & $\odot .002$ \\
\hline 18: & 2 & 1 & 1 & 1 & 1 & 3 & 1 & 0 & 1 & 0 & 1 & 2 & 9910.8895 & -0.0029 & 0.002 \\
\hline 19: & 2 & 1 & 1 & 1 & 1 & 1 & 1 & $\odot$ & 1 & 0 & 1 & $\odot$ & 9913.4174 & $\odot . \odot \odot \odot \odot$ & $\odot .0 \odot 2$ \\
\hline 20: & 2 & 1 & 1 & 1 & 3 & 2 & 1 & $\odot$ & 1 & 0 & 1 & 2 & 9913.8526 & $-\odot . \odot \odot 42$ & $\odot .0 \odot 2$ \\
\hline 21: & 2 & 1 & 1 & 1 & 1 & 2 & 1 & 0 & 1 & 0 & 1 & 1 & 9916.0712 & 0.0005 & $\odot .002$ \\
\hline 22: & 2 & 1 & 1 & 1 & 3 & 4 & 1 & 0 & 1 & 0 & 3 & 3 & 9917 & 18 & 02 \\
\hline 23: & 2 & 1 & 1 & 1 & 3 & 5 & 1 & 0 & 1 & 0 & 3 & 4 & 991 & 04 & 02 \\
\hline 24: & 2 & 1 & 1 & 1 & 2 & 2 & 1 & 0 & 1 & 0 & 0 & 1 & 99 & 15 & $\odot \odot 2$ \\
\hline 25: & 2 & 1 & 1 & 1 & 3 & 1 & 1 & $\odot$ & 1 & $\odot$ & 3 & 2 & 991 & $-\odot$. & 0.002 \\
\hline 26: & 2 & 1 & 1 & 1 & 2 & 3 & 1 & $\odot$ & 1 & 0 & 2 & 2 & 9920 & 11 & $\odot . \odot \odot 2$ \\
\hline 27: & 2 & 1 & 1 & 1 & 2 & 4 & 1 & 0 & 1 & 0 & 2 & 3 & 9920. & $-\odot$. & 0.002 \\
\hline 28: & 2 & 1 & 1 & 1 & 3 & 3 & 1 & $\odot$ & 1 & 0 & 3 & 3 & 9920.5515 & ๑०८ & 0.002 \\
\hline 29: & 2 & 1 & 1 & $\odot$ & 3 & 4 & 1 & $\odot$ & 1 & 1 & 3 & 4 & 9920.7543 & $-\odot . \odot \odot 22$ & ๑. $\odot \odot 2$ \\
\hline 30: & 2 & 1 & 1 & 1 & $\odot$ & 2 & 1 & $\odot$ & 1 & 0 & 2 & 1 & 9921.2748 & $-\odot . \odot \odot 21$ & ๑. $\odot \odot 2$ \\
\hline 31: & 2 & 1 & 1 & 1 & 1 & 2 & 1 & $\odot$ & 1 & 0 & 3 & 2 & 9922.3972 & $-\odot . \odot \odot 34$ & $\odot .0 \odot 2$ \\
\hline 32: & 2 & 2 & $\odot$ & $\odot$ & 3 & 3 & 1 & 1 & $\odot$ & 1 & 3 & 3 & 11120.9654 & $-\odot .0 \odot \odot 2$ & $0.0 \odot 2$ \\
\hline 33: & 2 & 2 & $\odot$ & $\odot$ & 0 & 2 & 1 & 1 & $\odot$ & 1 & 2 & 1 & 11121. & 0.0010 & 0.002 \\
\hline 34: & 2 & 2 & $\odot$ & $\odot$ & 3 & 3 & 1 & 1 & 0 & 1 & 1 & 2 & 111 & 07 & $0 \odot 2$ \\
\hline 35: & 2 & 2 & 0 & $\odot$ & 0 & 2 & 1 & 1 & $\odot$ & 1 & 2 & 2 & 3 & & 02 \\
\hline 36: & 2 & 2 & 0 & $\odot$ & 1 & 1 & 1 & 1 & $\odot$ & 1 & 1 & $\odot$ & 4 & & .002 \\
\hline 37: & 2 & 2 & 0 & $\odot$ & 3 & 4 & 1 & 1 & $\odot$ & 1 & 3 & 3 & 11122. & 11 & 0.002 \\
\hline 38: & 2 & 2 & 0 & $\odot$ & 3 & 4 & 1 & 1 & $\odot$ & 1 & 3 & 4 & 1112 & 04 & $\odot .0 \odot 2$ \\
\hline 39: & 2 & 2 & 0 & $\odot$ & 1 & 1 & 1 & 1 & $\odot$ & 1 & 1 & 1 & 11123. & $0 \odot 7$ & 0.002 \\
\hline 40: & 2 & 2 & 0 & $\odot$ & 2 & 1 & 1 & 1 & $\odot$ & 1 & 2 & 1 & 11126.5789 & 0.0032 & 0.002 \\
\hline 41: & 2 & 2 & $\odot$ & $\odot$ & 3 & 2 & 1 & 1 & $\odot$ & 1 & 3 & 3 & 11126.5789 & $-\odot .0 \odot 29$ & 0.002 \\
\hline 42: & 2 & 2 & $\odot$ & $\odot$ & 3 & 2 & 1 & 1 & $\odot$ & 1 & 1 & 2 & 11126.8982 & $-\odot .0021$ & 0.002 \\
\hline 43: & 2 & 2 & $\odot$ & $\odot$ & 2 & 3 & 1 & 1 & $\odot$ & 1 & 2 & 2 & 11127.2961 & 0.0016 & $\odot .0 \odot 2$ \\
\hline 44: & 2 & 2 & 0 & $\odot$ & 2 & 3 & 1 & 1 & $\odot$ & 1 & 2 & 3 & 11127.5291 & $-\odot .0 \odot \odot 6$ & $\odot .0 \odot 2$ \\
\hline 45: & 2 & 2 & 0 & $\odot$ & 2 & 4 & 1 & 1 & $\odot$ & 1 & 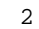 & 3 & 11127.5372 & 0.0031 & 0.002 \\
\hline 46: & 2 & 2 & 0 & $\odot$ & 1 & 3 & 1 & 1 & $\odot$ & 1 & 3 & 3 & 1112 & $-\odot$ & 0.002 \\
\hline 47: & 2 & 2 & 0 & $\odot$ & 3 & 2 & 1 & 1 & 0 & 1 & & 1 & & & $\frac{9}{2}$ \\
\hline 48: & 2 & 2 & $\odot$ & 0 & 1 & 3 & 1 & 1 & $\odot$ & 1 & 1 & 2 & 11 & $-\odot$ & $\odot .0 \odot 2$ \\
\hline 49: & 2 & 2 & $\odot$ & $\odot$ & 1 & 3 & 1 & 1 & $\odot$ & 1 & 3 & 2 & 1113 & 19 & $\odot .0 \odot 2$ \\
\hline 50: & 2 & 2 & 0 & $\odot$ & 3 & 5 & 1 & 1 & $\odot$ & 1 & 3 & 4 & 11 & 14 & $\odot .0 \odot 2$ \\
\hline 51: & 2 & 2 & 0 & $\odot$ & 1 & 2 & 1 & 1 & $\odot$ & 1 & 1 & 1 & 111 & $-\odot$ & $\odot .002$ \\
\hline 52: & 2 & 2 & 0 & $\odot$ & 2 & 2 & 1 & 1 & $\odot$ & 1 & $\odot$ & 1 & 11133.8787 & -0. & 0.002 \\
\hline 53: & 2 & 2 & $\odot$ & $\odot$ & 1 & 2 & 1 & 1 & $\odot$ & 1 & 3 & 2 & 11134.3954 & -0.0010 & 0.002 \\
\hline 54: & 2 & 2 & $\odot$ & $\odot$ & 3 & 1 & 1 & 1 & $\odot$ & 1 & 3 & 2 & 11135.6758 & -0.0036 & 0.002 \\
\hline 55: & 2 & 2 & $\odot$ & 1 & 3 & 3 & 1 & 1 & $\odot$ & $\odot$ & 3 & 3 & 11133.3062 & $-\odot .0006$ & $\odot .0 \odot 2$ \\
\hline 56: & 2 & 2 & $\odot$ & 1 & 0 & 2 & 1 & 1 & $\odot$ & $\odot$ & 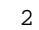 & 1 & 11133.5964 & -0. & $\odot .0 \odot 2$ \\
\hline 57: & 2 & 2 & $\odot$ & 1 & 3 & 3 & 1 & 1 & $\odot$ & 0 & 1 & 2 & 11133. & $-\odot$ & 0.002 \\
\hline 58: & 2 & 2 & 0 & 1 & 0 & 2 & 1 & 1 & 0 & 0 & ? & 2 & 1113 & $\odot$. & .002 \\
\hline 59: & 2 & 2 & $\odot$ & 1 & 3 & 4 & 1 & 1 & $\odot$ & 0 & 3 & 3 & 4 & 12 & 2 \\
\hline 60: & 2 & 2 & $\odot$ & 1 & 3 & 4 & 1 & 1 & 0 & $\odot$ & & 4 & & & $\odot .0 \odot 2$ \\
\hline 61: & 2 & 2 & 0 & 1 & 1 & 1 & 1 & 1 & $\odot$ & 0 & & 1 & & -0 & $\odot .0 \odot 2$ \\
\hline 62: & 2 & 2 & 0 & 1 & 2 & 1 & 1 & 1 & $\odot$ & 0 & 2 & 1 & 11 & $\odot 2$ & $\odot .0 \odot 2$ \\
\hline 63: & 2 & 2 & 0 & 1 & 2 & 3 & 1 & 1 & $\odot$ & $\odot$ & 2 & 2 & 11 & -0 & $\odot .0 \odot 2$ \\
\hline 64: & 2 & 2 & 0 & 1 & 2 & 1 & 1 & 1 & $\odot$ & 0 & 2 & 2 & 111 & 60 & 0.002 \\
\hline 65: & 2 & 2 & 0 & 1 & 2 & 3 & 1 & 1 & 0 & $\theta$ & 2 & 3 & 111 & 72 & $\odot .0 \odot 2$ \\
\hline 66: & 2 & 2 & 0 & 1 & 2 & 4 & 1 & 1 & $\odot$ & 0 & 2 & 3 & 11139.8791 & 0.0030 & 0.002 \\
\hline 67: & 2 & 2 & $\odot$ & 1 & 3 & 5 & 1 & 1 & $\odot$ & 0 & 3 & 4 & 11143.1265 & $\odot .0 \odot \odot 7$ & $\odot .0 \odot 2$ \\
\hline 68: & 2 & 2 & 1 & $\odot$ & $\odot$ & 2 & 1 & 1 & 1 & 1 & 2 & 2 & $11576.67 \odot 2$ & 912 & $\odot .0 \odot 2$ \\
\hline 69: & 2 & 2 & 1 & $\odot$ & 3 & 2 & 1 & 1 & 1 & 1 & 1 & 1 & 11578.5232 & $-\odot$ & $\odot .0 \odot 2$ \\
\hline 70: & 2 & 2 & 1 & $\odot$ & 1 & 2 & 1 & 1 & 1 & 1 & 3 & 2 & 1158 & $-\odot$ & 0.002 \\
\hline 71: & 2 & 2 & 1 & 0 & 3 & 3 & 1 & 1 & 1 & 1 & 1 & 2 & 115 & -6 & 0.002 \\
\hline 72: & 2 & 2 & 1 & $\odot$ & 2 & 2 & 1 & 1 & 1 & 1 & $\odot$ & 1 & 115 & -0.0006 & $0.0 \odot 2$ \\
\hline 73: & 2 & 2 & 1 & 0 & 3 & 1 & 1 & 1 & 1 & 1 & 3 & 2 & 11584.9247 & $-\odot . \odot \odot 49$ & 0.002 \\
\hline 74: & 2 & 2 & 1 & $\odot$ & 2 & 4 & 1 & 1 & 1 & 1 & 2 & 3 & 1158 & & $\odot .0 \odot 2$ \\
\hline 75: & 2 & 2 & 1 & $\odot$ & 2 & 3 & 1 & 1 & 1 & 1 & 2 & 3 & 11586 & -0 & $0.0 \odot 2$ \\
\hline 76: & 2 & 2 & 1 & 0 & 2 & 3 & 1 & 1 & 1 & 1 & 2 & 2 & 11586.9222 & 945 & 0.002 \\
\hline 77: & 2 & 2 & 1 & $\odot$ & 3 & 5 & 1 & 1 & 1 & 1 & 3 & 4 & 11587.8557 & $-\odot . \odot \odot 23$ & $\odot .0 \odot 2$ \\
\hline 78: & 2 & 2 & 1 & $\odot$ & 3 & 3 & 1 & 1 & 1 & 1 & 3 & 3 & 11588.1 & -0. & $\odot .0 \odot 2$ \\
\hline 79: & 2 & 2 & 1 & $\odot$ & 1 & 2 & 1 & 1 & 1 & 1 & 1 & 1 & 11589.4638 & $\odot .0021$ & $\odot .0 \odot 2$ \\
\hline 80: & 2 & 2 & 1 & $\odot$ & 3 & 4 & 1 & 1 & 1 & 1 & 3 & 3 & 11590.8741 & 0.0014 & $\odot .0 \odot 2$ \\
\hline 81: & 2 & 2 & 1 & $\odot$ & 3 & 2 & 1 & 1 & 1 & 1 & 1 & 2 & 11593.5913 & $-\odot .0 \odot 38$ & $\odot .0 \odot 2$ \\
\hline
\end{tabular}




\begin{tabular}{|c|c|c|c|c|c|c|c|c|c|c|c|}
\hline $82 /$ & 2 & 2 & 1 & 0 & 1 & 3 & 1 & 1 & 1 & 1 & 1 \\
\hline 83: & 2 & 2 & 1 & $\odot$ & 3 & 2 & 1 & 1 & 1 & 1 & 3 \\
\hline 84: & 2 & 2 & 1 & $\odot$ & 1 & 3 & 1 & 1 & 1 & 1 & 3 \\
\hline 85: & 2 & 2 & 1 & 1 & 2 & 1 & 1 & 1 & 1 & $\odot$ & $\odot$ \\
\hline 86: & 2 & 2 & 1 & 1 & 3 & 4 & 1 & 1 & 1 & $\odot$ & 3 \\
\hline 87: & 2 & 2 & 1 & 1 & 1 & 3 & 1 & 1 & 1 & $\odot$ & 3 \\
\hline 88: & 2 & 2 & 1 & 1 & $\odot$ & 2 & 1 & 1 & 1 & $\odot$ & 2 \\
\hline 89: & 2 & 2 & 1 & 1 & 3 & 3 & 1 & 1 & 1 & $\odot$ & 1 \\
\hline $90:$ & 2 & 2 & 1 & 1 & 2 & 2 & 1 & 1 & 1 & $\odot$ & $\odot$ \\
\hline 91: & 2 & 2 & 1 & 1 & 2 & 2 & 1 & 1 & 1 & $\odot$ & $\odot$ \\
\hline 92: & 2 & 2 & 1 & 1 & 2 & 4 & 1 & 1 & 1 & $\odot$ & 2 \\
\hline 93/ & 2 & 2 & 1 & 1 & 2 & 3 & 1 & 1 & 1 & $\odot$ & 2 \\
\hline 94: & 2 & 2 & 1 & 1 & 2 & 3 & 1 & 1 & 1 & 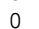 & 2 \\
\hline 95: & 2 & 2 & 1 & 1 & 3 & 5 & 1 & 1 & 1 & $\odot$ & 3 \\
\hline 96: & 2 & 2 & 1 & 1 & 3 & 3 & 1 & 1 & 1 & $\odot$ & 3 \\
\hline 97: & 2 & 2 & 1 & 1 & 1 & 2 & 1 & 1 & 1 & $\odot$ & 1 \\
\hline 98: & 2 & 2 & 1 & 1 & 2 & 2 & 1 & 1 & 1 & $\odot$ & 2 \\
\hline 99: & 2 & 2 & 1 & 1 & $\odot$ & 2 & 1 & 1 & 1 & $\odot$ & 2 \\
\hline 100: & 2 & 2 & 1 & 1 & 3 & 4 & 1 & 1 & 1 & 0 & 3 \\
\hline 101: & 3 & 1 & 2 & $\odot$ & $\odot$ & 3 & 2 & $\odot$ & 2 & 1 & 2 \\
\hline 102: & 3 & 1 & 2 & $\odot$ & 1 & 3 & 2 & $\odot$ & 2 & 1 & 1 \\
\hline 103: & 3 & 1 & 2 & $\odot$ & 3 & 6 & 2 & $\odot$ & 2 & 1 & 3 \\
\hline 104: & 3 & 1 & 2 & $\odot$ & 1 & 4 & 2 & $\odot$ & 2 & 1 & 1 \\
\hline 105: & 3 & 1 & 2 & $\odot$ & 2 & 4 & 2 & $\odot$ & 2 & 1 & 2 \\
\hline 106: & 3 & 1 & 2 & $\odot$ & 2 & 5 & 2 & $\odot$ & 2 & 1 & 2 \\
\hline 107: & 3 & 1 & 2 & $\odot$ & 3 & 5 & 2 & $\odot$ & 2 & 1 & 3 \\
\hline 108: & 3 & 1 & 2 & $\odot$ & 2 & 3 & 2 & $\odot$ & 2 & 1 & $\odot$ \\
\hline 109: & 3 & 1 & 2 & 1 & 1 & 3 & 2 & $\odot$ & 2 & $\odot$ & 1 \\
\hline 110: & 3 & 1 & 2 & 1 & $\odot$ & 3 & 2 & $\odot$ & 2 & $\odot$ & 2 \\
\hline 111: & 3 & 1 & 2 & 1 & 1 & 3 & 2 & $\odot$ & 2 & 0 & 1 \\
\hline 112: & 3 & 1 & 2 & 1 & 3 & 6 & 2 & 0 & 2 & $\odot$ & 3 \\
\hline 113: & 3 & 1 & 2 & 1 & 3 & 3 & 2 & $\odot$ & 2 & $\odot$ & 3 \\
\hline 114: & 3 & 1 & 2 & 1 & 1 & 4 & 2 & $\odot$ & 2 & 0 & 1 \\
\hline 115: & 3 & 1 & 2 & 1 & 2 & 5 & 2 & $\odot$ & 2 & $\odot$ & 2 \\
\hline 116: & 3 & 1 & 2 & 1 & 3 & 5 & 2 & $\odot$ & 2 & $\odot$ & 3 \\
\hline 117: & 3 & 1 & 2 & 1 & 1 & 2 & 2 & $\odot$ & 2 & $\odot$ & 1 \\
\hline 118: & 3 & 1 & 2 & 1 & 3 & 3 & 2 & $\odot$ & 2 & 0 & 3 \\
\hline 119: & 3 & 1 & 2 & 1 & 2 & 3 & 2 & $\odot$ & 2 & 0 & 0 \\
\hline $120:$ & 3 & 1 & 2 & 1 & 3 & 4 & 2 & $\odot$ & 2 & $\odot$ & 3 \\
\hline 121: & 3 & 1 & 2 & 1 & 3 & 4 & 2 & $\odot$ & 2 & $\odot$ & 3 \\
\hline 122: & 3 & 1 & 2 & 1 & 3 & 5 & 2 & $\odot$ & 2 & $\odot$ & 3 \\
\hline 123: & 3 & 2 & 1 & $\odot$ & 3 & 3 & 2 & 1 & 1 & 1 & 3 \\
\hline 124: & 3 & 2 & 1 & $\odot$ & 3 & 5 & 2 & 1 & 1 & 1 & 3 \\
\hline 125: & 3 & 2 & 1 & $\odot$ & 2 & 3 & 2 & 1 & 1 & 1 & 2 \\
\hline 126: & 3 & 2 & 1 & $\odot$ & 1 & 2 & 2 & 1 & 1 & 1 & 1 \\
\hline 127: & 3 & 2 & 1 & $\odot$ & 3 & 4 & 2 & 1 & 1 & 1 & 3 \\
\hline 128: & 3 & 2 & 1 & $\odot$ & 2 & 5 & 2 & 1 & 1 & 1 & 2 \\
\hline 129: & 3 & 2 & 1 & $\odot$ & 3 & 6 & 2 & 1 & 1 & 1 & 3 \\
\hline 130: & 3 & 2 & 1 & $\odot$ & 1 & 4 & 2 & 1 & 1 & 1 & 1 \\
\hline 131: & 3 & 2 & 1 & 0 & 1 & 3 & 2 & 1 & 1 & 1 & 1 \\
\hline 132: & 3 & 2 & 1 & $\odot$ & 3 & 3 & 2 & 1 & 1 & 1 & 3 \\
\hline 133: & 3 & 2 & 1 & $\odot$ & 3 & 1 & 2 & 1 & 1 & 1 & 3 \\
\hline 134: & 3 & 2 & 1 & 1 & 3 & 5 & 2 & 1 & 1 & $\odot$ & 3 \\
\hline 135: & 3 & 2 & 1 & 1 & 2 & 3 & 2 & 1 & 1 & $\odot$ & 2 \\
\hline 136: & 3 & 2 & 1 & 1 & 2 & 5 & 2 & 1 & 1 & 0 & 2 \\
\hline 137: & 3 & 2 & 1 & 1 & 3 & 6 & 2 & 1 & 1 & $\odot$ & 3 \\
\hline 138: & 3 & 2 & 1 & 1 & 1 & 4 & 2 & 1 & 1 & $\odot$ & 1 \\
\hline 139: & 3 & 2 & 1 & 1 & $\odot$ & 3 & 2 & 1 & 1 & $\odot$ & $\odot$ \\
\hline 140: & 3 & 2 & 1 & 1 & 1 & 3 & 2 & 1 & 1 & $\odot$ & 1 \\
\hline 141: & 3 & 2 & 2 & $\odot$ & 3 & 3 & 2 & 1 & 2 & 1 & 1 \\
\hline 142: & 3 & 2 & 2 & $\odot$ & $\odot$ & 3 & 2 & 1 & 2 & 1 & 0 \\
\hline 143: & 3 & 2 & 2 & $\odot$ & 3 & 6 & 2 & 1 & 2 & 1 & 3 \\
\hline 144: & 3 & 2 & 2 & $\odot$ & 3 & 4 & 2 & 1 & 2 & 1 & 1 \\
\hline 145: & 3 & 2 & 2 & $\odot$ & 2 & 5 & 2 & 1 & 2 & 1 & 2 \\
\hline 146: & 3 & 2 & 2 & $\odot$ & 3 & 5 & 2 & 1 & 2 & 1 & 3 \\
\hline $147 /$ & 3 & 2 & 2 & $\odot$ & 1 & 4 & 2 & 1 & 2 & 1 & 3 \\
\hline 148: & 3 & 2 & 2 & 1 & 2 & 3 & 2 & 1 & 2 & $\odot$ & $\odot$ \\
\hline 149: & 3 & 2 & 2 & 1 & 3 & 6 & 2 & 1 & 2 & $\odot$ & 3 \\
\hline 150: & 3 & 2 & 2 & 1 & 2 & 4 & 2 & 1 & 2 & $\odot$ & 2 \\
\hline 151: & 3 & 2 & 2 & 1 & 2 & 5 & 2 & 1 & 2 & $\odot$ & 2 \\
\hline 152: & 3 & 2 & 2 & 1 & 3 & 5 & 2 & 1 & 2 & $\odot$ & 3 \\
\hline 153: & 3 & 2 & 2 & 1 & $\odot$ & 3 & 2 & 1 & 2 & $\odot$ & 2 \\
\hline 154/ & 3 & 2 & 2 & 1 & 1 & 4 & 2 & 1 & 2 & 0 & 3 \\
\hline 155: & 3 & 3 & $\odot$ & $\odot$ & $\odot$ & 3 & 2 & 2 & 0 & 1 & 0 \\
\hline 156: & 3 & 3 & 0 & $\odot$ & 3 & 5 & 2 & 2 & $\odot$ & 1 & 3 \\
\hline 157: & 3 & 3 & $\odot$ & $\odot$ & 2 & 5 & 2 & 2 & $\odot$ & 1 & 2 \\
\hline 158: & 3 & 3 & $\odot$ & $\odot$ & 3 & 6 & 2 & 2 & $\odot$ & 1 & 3 \\
\hline 159: & 3 & 3 & $\odot$ & $\odot$ & 2 & 3 & 2 & 2 & $\odot$ & 1 & 2 \\
\hline 160: & 3 & 3 & $\odot$ & 1 & 3 & 5 & 2 & 2 & $\odot$ & $\odot$ & 3 \\
\hline 161: & 3 & 3 & 0 & 1 & 3 & 4 & 2 & 2 & 0 & $\odot$ & 3 \\
\hline 162: & 3 & 3 & $\odot$ & 1 & 3 & 4 & 2 & 2 & $\odot$ & $\odot$ & 3 \\
\hline 163: & 3 & 3 & $\odot$ & 1 & 3 & 3 & 2 & 2 & $\odot$ & $\odot$ & 3 \\
\hline 164: & 3 & 3 & $\odot$ & 1 & $\odot$ & 3 & 2 & 2 & $\odot$ & 0 & 0 \\
\hline 165: & 3 & 3 & $\odot$ & 1 & 1 & 2 & 2 & 2 & $\odot$ & $\odot$ & 1 \\
\hline 166: & 3 & 3 & $\odot$ & 1 & 3 & 5 & 2 & 2 & $\odot$ & $\odot$ & 3 \\
\hline 167: & 3 & 3 & 0 & 1 & 1 & 4 & 2 & 2 & 0 & $\odot$ & 1 \\
\hline 168: & 3 & 3 & $\odot$ & 1 & 2 & 5 & 2 & 2 & $\odot$ & $\odot$ & 2 \\
\hline
\end{tabular}




$\begin{array}{lllllll}169: & 3 & 3 & 0 & 1 & 3 & 3 \\ 170: & 3 & 3 & 0 & 1 & 3 & 6 \\ 171: & 3 & 3 & 0 & 1 & 1 & 3 \\ 172: & 3 & 3 & 0 & 1 & 3 & 2 \\ 173: & 3 & 3 & 1 & 0 & 3 & 4 \\ 174: & 3 & 3 & 1 & 0 & 1 & 4 \\ 175: & 3 & 3 & 1 & 0 & 0 & 3 \\ 176: & 3 & 3 & 1 & 0 & 2 & 2 \\ 177: & 3 & 3 & 1 & 0 & 2 & 4 \\ 178: & 3 & 3 & 1 & 0 & 2 & 5 \\ 179: & 3 & 3 & 1 & 0 & 1 & 3 \\ 180: & 3 & 3 & 1 & 0 & 2 & 3 \\ 181: & 3 & 3 & 1 & 0 & 3 & 5 \\ 182: & 3 & 3 & 1 & 0 & 3 & 6 \\ 183: & 3 & 3 & 1 & 1 & 3 & 5 \\ 184: & 3 & 3 & 1 & 1 & 3 & 3 \\ 185: & 3 & 3 & 1 & 1 & 3 & 4 \\ 186: & 3 & 3 & 1 & 1 & 1 & 4 \\ 187: & 3 & 3 & 1 & 1 & 0 & 3 \\ 188: & 3 & 3 & 1 & 1 & 2 & 4 \\ 189: & 3 & 3 & 1 & 1 & 2 & 5 \\ 190: & 3 & 3 & 1 & 1 & 1 & 2 \\ 191: & 3 & 3 & 1 & 1 & 1 & 3 \\ 192: & 3 & 3 & 1 & 1 & 3 & 5 \\ 193: & 3 & 3 & 1 & 1 & 3 & 6 \\ 194: & 3 & 3 & 1 & 1 & 3 & 3\end{array}$

\begin{tabular}{|c|c|c|c|c|c|c|c|c|}
\hline 2 & 2 & $\odot$ & $\odot$ & 3 & 3 & 17320.4489 & $-\odot .0 \odot 64$ & $\odot . \odot \odot 2$ \\
\hline 2 & 2 & 0 & $\odot$ & 3 & 5 & 17323.1303 & 0.0042 & $\odot . \odot \odot 2$ \\
\hline 2 & 2 & $\odot$ & $\odot$ & 1 & 2 & 17323.7936 & $-\odot . \odot \odot 25$ & $\odot .0 \odot 2$ \\
\hline 2 & 2 & $\odot$ & $\odot$ & 3 & 2 & 17325.2663 & $-\odot . \odot \odot 73$ & $\odot .0 \odot 2$ \\
\hline 2 & 2 & 1 & 1 & 3 & 3 & 17579.1659 & $\odot .0 \odot 21$ & $\odot . \odot \odot 2$ \\
\hline 2 & 2 & 1 & 1 & 1 & 3 & 17580.2096 & -0.0003 & $\odot .002$ \\
\hline 2 & 2 & 1 & 1 & 0 & 2 & 17580.4060 & $\odot .0017$ & $\odot .002$ \\
\hline & 2 & 1 & 1 & 2 & 1 & 17581.3353 & -0.0048 & $\odot .0 \odot 2$ \\
\hline & 2 & 1 & 1 & 2 & 3 & 17581.3566 & $-\odot .0007$ & $\odot .0 \odot 2$ \\
\hline 2 & 2 & 1 & 1 & 2 & 4 & 17581.3641 & ๑. 0016 & $\odot . \odot \odot 2$ \\
\hline 2 & 2 & 1 & 1 & 1 & 2 & 17581.7352 & $-\odot . \odot \odot \odot 7$ & $\odot .0 \odot 2$ \\
\hline 2 & 2 & 1 & 1 & 2 & 2 & 17582.3216 & $-\odot .0038$ & 0.002 \\
\hline 2 & 2 & 1 & 1 & 3 & 4 & 17582.3422 & 0.0052 & 0.002 \\
\hline 2 & 2 & 1 & 1 & 3 & 5 & 17583.1195 & $\odot .0004$ & $\odot .0 \odot 2$ \\
\hline 2 & 2 & 1 & $\odot$ & 3 & 5 & 17580.9176 & -0.0061 & $\odot .0 \odot 2$ \\
\hline 2 & 2 & 1 & $\odot$ & 3 & 2 & 17588.0530 & -0.0015 & $\odot .0 \odot 2$ \\
\hline 2 & 2 & 1 & $\odot$ & 3 & 3 & 17591.4185 & $\odot .0028$ & $\odot . \odot \odot 2$ \\
\hline 2 & 2 & 1 & $\odot$ & 1 & 3 & 17592.4630 & 0.0008 & $\odot .002$ \\
\hline 2 & 2 & 1 & $\odot$ & 0 & 2 & 17592.6586 & $\odot .0023$ & 0.002 \\
\hline 2 & 2 & 1 & $\odot$ & 2 & 3 & 17593.6114 & 0.0019 & $\odot .002$ \\
\hline & 2 & 1 & $\Theta$ & 2 & 4 & 17593.6114 & -0.0031 & $\odot .0 \odot 2$ \\
\hline 2 & 2 & 1 & $\odot$ & 1 & 1 & 17593.6319 & $\odot .0025$ & $\odot .002$ \\
\hline & 2 & 1 & $\odot$ & 1 & 2 & 17593.9842 & $-0.0 \odot 4 \odot$ & $\odot .0 \odot 2$ \\
\hline & 2 & 1 & $\odot$ & 3 & 4 & 17594.5921 & $\odot .0031$ & $\odot . \odot \odot 2$ \\
\hline & 2 & 1 & $\odot$ & 3 & 5 & 17595.3749 & 0.0035 & $\odot .002$ \\
\hline & 2 & 1 & $\odot$ & 3 & 3 & 17599.0333 & $-\odot . \odot \odot \odot 4$ & $\odot .0 \odot 2$ \\
\hline
\end{tabular}


Table 3S. Transition frequencies of DCM-Ne(20) Cl(35)-Cl(37)

\begin{tabular}{|c|c|c|c|c|c|c|c|c|c|c|c|c|c|c|c|}
\hline & & & $K_{\mathrm{c}}{ }^{\prime}$ & $v^{\prime}$ & $I$ & $F^{\prime}$ & $J^{\prime \prime}$ & $K_{\mathrm{a}}^{\prime}$ & & $K_{c} "$ & $v^{\prime \prime}$ & $I^{\prime \prime}$ & $F^{\prime \prime}$ obs & o-c & error \\
\hline 1: & 2 & 1 & 1 & $\odot$ & 1 & 3 & 1 & $\odot$ & 1 & 1 & 1 & 2 & 10275.2399 & $\odot . \odot \odot 26$ & $\odot . \odot \odot 2$ \\
\hline 2: & 2 & 1 & 1 & $\odot$ & 3 & 2 & 1 & $\odot$ & 1 & 1 & 1 & 2 & 10277.9056 & -0.0014 & 0.002 \\
\hline 3: & 2 & 1 & 1 & $\odot$ & 1 & 2 & 1 & $\odot$ & 1 & 1 & 1 & 1 & 10279.4954 & $\odot .0028$ & $\odot .0 \odot 2$ \\
\hline 4: & 2 & 1 & 1 & $\odot$ & $\odot$ & 2 & 1 & $\odot$ & 1 & 1 & 1 & 1 & 10281.0379 & $\odot . \odot \odot 16$ & $\odot . \odot \odot 2$ \\
\hline 5: & 2 & 1 & 1 & $\odot$ & 3 & 4 & 1 & $\odot$ & 1 & 1 & 3 & 3 & 10281.4746 & $\odot . \odot \odot \odot 5$ & $\odot . \odot \odot 2$ \\
\hline 6: & 2 & 1 & 1 & $\odot$ & 3 & 5 & 1 & $\odot$ & 1 & 1 & 3 & 4 & 10281.8693 & $\odot .0023$ & $\odot . \odot \odot 2$ \\
\hline 7: & 2 & 1 & 1 & $\odot$ & 2 & 2 & 1 & $\odot$ & 1 & 1 & 2 & 1 & 10282.8362 & $-\odot .0011$ & $\odot .0 \odot 2$ \\
\hline 8: & 2 & 1 & 1 & $\odot$ & 2 & 4 & 1 & $\odot$ & 1 & 1 & 2 & 3 & 10283.7439 & $\odot .0 \odot 15$ & $\odot . \odot \odot 2$ \\
\hline 9: & 2 & 1 & 1 & $\odot$ & 2 & 3 & 1 & $\odot$ & 1 & 1 & 2 & 2 & 10283.8890 & $\odot . \odot \odot 13$ & $\odot . \odot \odot 2$ \\
\hline 10: & 2 & 1 & 1 & 0 & 3 & 3 & 1 & $\odot$ & 1 & 1 & 3 & 3 & 10284.0327 & $-\odot .0 \odot \odot 4$ & $\odot . \odot \odot 2$ \\
\hline 11: & 2 & 1 & 1 & $\odot$ & 0 & 2 & 1 & $\odot$ & 1 & 1 & 0 & 1 & 10285.3055 & $\odot .0 \odot 2 \odot$ & $\odot .0 \odot 2$ \\
\hline 12: & 2 & 1 & 1 & $\odot$ & 1 & 2 & 1 & $\odot$ & 1 & 1 & 3 & 2 & 10285.3183 & $\odot .0035$ & 0.002 \\
\hline 13: & 2 & 1 & 1 & 1 & 2 & 1 & 1 & $\odot$ & 1 & 0 & 2 & 1 & 10285.6244 & $-\odot .0010$ & $\odot .0 \odot 2$ \\
\hline 14: & 2 & 1 & 1 & 1 & 3 & 2 & 1 & 0 & 1 & 0 & 3 & 3 & 10286.0807 & -0.0005 & 0.002 \\
\hline 15: & 2 & 1 & 1 & 0 & 3 & 3 & 1 & $\odot$ & 1 & 1 & 1 & 2 & 10288.4941 & -0.0016 & 0.002 \\
\hline 16: & 2 & 1 & 1 & $\odot$ & 1 & 3 & 1 & $\odot$ & 1 & 1 & 3 & 2 & 10293.5812 & $\odot .0034$ & $\odot .002$ \\
\hline 17: & 2 & 1 & 1 & $\odot$ & 2 & 2 & 1 & $\odot$ & 1 & 1 & 2 & 2 & 10293.7985 & $\odot .0003$ & $\odot .002$ \\
\hline 18: & 2 & 1 & 1 & 0 & 3 & 4 & 1 & $\odot$ & 1 & 1 & 3 & 4 & 10295.1710 & $-\odot . \odot \odot 22$ & $\odot . \odot \odot 2$ \\
\hline 19: & 2 & 1 & 1 & $\odot$ & 3 & 2 & 1 & $\odot$ & 1 & 1 & 3 & 2 & 10296.2479 & $\odot .00 \odot 4$ & $\odot .002$ \\
\hline 20: & 2 & 1 & 1 & 1 & 0 & 2 & 1 & $\odot$ & 1 & 0 & 2 & 2 & 10287.1219 & $\odot .0018$ & $\odot .0 \odot 2$ \\
\hline 21: & 2 & 1 & 1 & 1 & 1 & 3 & 1 & $\odot$ & 1 & 0 & 1 & 2 & 10287.8751 & $\odot .0 \odot \odot 9$ & $\odot .002$ \\
\hline $22 /$ & 2 & 1 & 1 & 1 & 1 & 1 & 1 & $\odot$ & 1 & 0 & 1 & $\odot$ & 10290.4244 & $\odot .0065$ & $\odot .0 \odot 2$ \\
\hline 23: & 2 & 1 & 1 & 1 & 3 & 2 & 1 & $\odot$ & 1 & $\odot$ & 1 & 2 & 10290.5426 & $-\odot . \odot \odot 12$ & $\odot . \odot \odot 2$ \\
\hline 24: & 2 & 1 & 1 & 1 & 1 & 2 & 1 & $\odot$ & 1 & 0 & 1 & 1 & 10292.1255 & $-\odot .0 \odot 39$ & $\odot .0 \odot 2$ \\
\hline 25: & 2 & 1 & 1 & 1 & 0 & 2 & 1 & $\odot$ & 1 & 0 & 1 & 1 & 10293.6721 & -0.0010 & $\odot .002$ \\
\hline 26: & 2 & 1 & 1 & 1 & 3 & 5 & 1 & 0 & 1 & 0 & 3 & 4 & 10294.5049 & $\odot .0011$ & 0.002 \\
\hline 27: & 2 & 1 & 1 & 1 & 2 & 2 & 1 & 0 & 1 & 0 & 2 & 1 & 10295.4728 & -0.0013 & $\odot .001$ \\
\hline 28: & 2 & 1 & 1 & 1 & 3 & 1 & 1 & $\odot$ & 1 & 0 & 3 & 2 & 10296.0468 & -0.0037 & $\odot . \odot \odot 2$ \\
\hline 29: & 2 & 1 & 1 & 1 & 2 & 4 & 1 & $\odot$ & 1 & 0 & 2 & 3 & 10296.3795 & $\odot .0003$ & $\odot .0 \odot 2$ \\
\hline 30: & 2 & 1 & 1 & 1 & 2 & 3 & 1 & $\odot$ & 1 & 0 & 2 & 2 & 10296.5241 & $-\odot . \odot \odot \odot 4$ & $\odot . \odot \odot 2$ \\
\hline 31: & 2 & 1 & 1 & 1 & 3 & 3 & 1 & $\odot$ & 1 & 0 & 3 & 3 & 10296.6732 & $\odot .0032$ & $\odot .002$ \\
\hline 32: & 2 & 1 & 1 & 1 & 1 & 2 & 1 & $\odot$ & 1 & 0 & 3 & 2 & 10297.9543 & 0.0026 & 0.002 \\
\hline 33: & 2 & 1 & 1 & 1 & 0 & 2 & 1 & $\odot$ & 1 & 0 & 0 & 1 & 10297.9389 & $-\odot .0014$ & 0.002 \\
\hline 34: & 2 & 1 & 1 & 1 & 0 & 2 & 1 & $\odot$ & 1 & 0 & 3 & 2 & 10299.4956 & $\odot .00 \odot 2$ & $\odot .002$ \\
\hline 35: & 2 & 1 & 1 & 1 & 3 & 3 & 1 & $\odot$ & 1 & 0 & 1 & 2 & 10301.1323 & $-\odot . \odot \odot \odot 2$ & $\odot . \odot \odot 2$ \\
\hline 36: & 2 & 1 & 1 & 1 & 3 & 2 & 1 & 0 & 1 & 0 & 1 & 1 & 10303.0582 & $-\odot .0 \odot 39$ & $\odot . \odot \odot 2$ \\
\hline 37: & 2 & 1 & 1 & 1 & 1 & 3 & 1 & $\odot$ & 1 & 0 & 3 & 2 & 10306.2140 & $-\odot . \odot \odot \odot 6$ & $\odot .001$ \\
\hline 38: & 2 & 1 & 1 & 1 & 2 & 2 & 1 & 0 & 1 & 0 & 2 & 2 & 10306.4350 & $\odot .0000$ & 0.002 \\
\hline 39: & 2 & 1 & 1 & 1 & 2 & 1 & 1 & 0 & 1 & 0 & 0 & 1 & 10307.4043 & -0.0023 & 0.002 \\
\hline 40: & 2 & 1 & 1 & 1 & 3 & 4 & 1 & $\odot$ & 1 & 0 & 3 & 4 & 10307.8075 & $-0.0 \odot 25$ & $\odot .0 \odot 2$ \\
\hline 41: & 2 & 1 & 1 & 1 & 3 & 2 & 1 & $\odot$ & 1 & 0 & 3 & 2 & 10308.8826 & -0.0017 & $\odot . \odot \odot 2$ \\
\hline 42: & 2 & 2 & $\odot$ & $\odot$ & 3 & 3 & 1 & 1 & $\odot$ & 1 & 3 & 3 & 11097.1311 & $\odot . \odot \odot \odot \odot$ & $\odot .0 \odot 2$ \\
\hline 43: & 2 & 2 & $\odot$ & $\odot$ & $\odot$ & 2 & 1 & 1 & $\odot$ & 1 & 2 & 1 & 11097.3084 & 0.0005 & $\odot .0 \odot 2$ \\
\hline 44: & 2 & 2 & 0 & 0 & 3 & 3 & 1 & 1 & $\odot$ & 1 & 1 & 2 & 11097.4354 & 0.0015 & 0.002 \\
\hline 45: & 2 & 2 & 0 & 0 & 1 & 1 & 1 & 1 & 0 & 1 & 1 & $\odot$ & 11097.9355 & $\odot .0 \odot 29$ & 0.002 \\
\hline 46: & 2 & 2 & 0 & 0 & 3 & 4 & 1 & 1 & 0 & 1 & 3 & 3 & 11098.0155 & $\odot .0011$ & 0.002 \\
\hline 47: & 2 & 2 & $\odot$ & $\odot$ & 3 & 4 & 1 & 1 & $\odot$ & 1 & 3 & 4 & 11099.1965 & $-\odot . \odot \odot \odot 4$ & $\odot . \odot \odot 2$ \\
\hline 48: & 2 & 2 & $\odot$ & $\odot$ & 1 & 1 & 1 & 1 & $\odot$ & 1 & 1 & 1 & 11099.4302 & $\odot . \odot \odot \odot 7$ & $\odot .0 \odot 2$ \\
\hline 49: & 2 & 2 & 0 & $\odot$ & 2 & 1 & 1 & 1 & $\odot$ & 1 & 2 & 1 & 11100.8136 & $-\odot .0 \odot \odot 8$ & $\odot .0 \odot 2$ \\
\hline 50: & 2 & 2 & 0 & 0 & 3 & 2 & 1 & 1 & 0 & 1 & 1 & 2 & 11101.1185 & -0.0019 & 0.002 \\
\hline 51: & 2 & 2 & 0 & 0 & 2 & 3 & 1 & 1 & 0 & 1 & 2 & 2 & 11101.4893 & $\odot .0017$ & 0.002 \\
\hline 52: & 2 & 2 & 0 & 0 & 2 & 3 & 1 & 1 & 0 & 1 & 2 & 3 & 11101.6529 & -0.0013 & $\odot .002$ \\
\hline 53: & 2 & 2 & $\odot$ & 0 & 2 & 4 & 1 & 1 & $\odot$ & 1 & 2 & 3 & 11101.7104 & $\odot .0011$ & $\odot .002$ \\
\hline 54: & 2 & 2 & $\odot$ & $\odot$ & 1 & 3 & 1 & 1 & $\odot$ & 1 & 1 & 2 & 11102.0768 & $\odot .0013$ & $\odot .0 \odot 2$ \\
\hline 55: & 2 & 2 & $\odot$ & 0 & 3 & 2 & 1 & 1 & $\odot$ & 1 & 1 & 1 & 11102.1005 & -0.0013 & $\odot . \odot \odot 2$ \\
\hline 56: & 2 & 2 & 0 & 0 & 3 & 2 & 1 & 1 & $\odot$ & 1 & 3 & 2 & 11102.6159 & -0.0018 & $\odot .0 \odot 2$ \\
\hline 57: & 2 & 2 & 0 & 0 & 1 & 3 & 1 & 1 & 0 & 1 & 3 & 2 & 11103.5743 & 0.0016 & 0.002 \\
\hline 58: & 2 & 2 & 0 & 0 & 3 & 5 & 1 & 1 & 0 & 1 & 3 & 4 & 11103.8620 & $\odot .00 \odot 8$ & 0.002 \\
\hline 59: & 2 & 2 & $\odot$ & 0 & 2 & 2 & 1 & 1 & $\odot$ & 1 & 1 & 1 & 11105.4310 & -0.0003 & $\odot . \odot \odot 2$ \\
\hline 60: & 2 & 2 & $\odot$ & $\odot$ & 1 & 2 & 1 & 1 & $\odot$ & 1 & 2 & 2 & 11105.5009 & $\odot . \odot \odot 23$ & $\odot .0 \odot 2$ \\
\hline 61: & 2 & 2 & $\odot$ & $\odot$ & 3 & 1 & 1 & 1 & $\odot$ & 1 & 1 & 2 & 11105.8086 & $-\odot .0 \odot 39$ & $\odot .0 \odot 2$ \\
\hline 62: & 2 & 2 & 0 & 0 & 2 & 2 & 1 & 1 & $\odot$ & 1 & 0 & 1 & 11105.8318 & $\odot .0003$ & 0.002 \\
\hline 63: & 2 & 2 & 0 & 0 & 1 & 2 & 1 & 1 & 0 & 1 & 1 & 1 & 11106.1378 & $\odot .0000$ & 0.002 \\
\hline 64: & 2 & 2 & 0 & 0 & 1 & 2 & 1 & 1 & $\odot$ & 1 & 0 & 1 & 11106.5366 & -0.0012 & $\odot .002$ \\
\hline 65: & 2 & 2 & $\odot$ & $\odot$ & 1 & 2 & 1 & 1 & $\odot$ & 1 & 3 & 2 & 11106.6533 & $-\odot . \odot \odot \odot 2$ & $\odot . \odot \odot 2$ \\
\hline 66: & 2 & 2 & $\odot$ & 0 & 3 & 1 & 1 & 1 & $\odot$ & 1 & 3 & 2 & 11107.3056 & -0.0042 & $\odot . \odot \odot 2$ \\
\hline 67: & 2 & 2 & $\odot$ & 1 & 3 & 3 & 1 & 1 & $\odot$ & 0 & 3 & 3 & 11109.9466 & $\odot . \odot \odot \odot 2$ & $\odot .002$ \\
\hline 68: & 2 & 2 & 0 & 1 & $\odot$ & 2 & 1 & 1 & $\odot$ & 0 & 2 & 1 & 11110.1245 & $\odot .0013$ & $\odot .002$ \\
\hline 69: & 2 & 2 & 0 & 1 & 3 & 3 & 1 & 1 & 0 & 0 & 1 & 2 & 11110.2493 & $\odot .0 \odot \odot 2$ & 0.002 \\
\hline 70: & 2 & 2 & 0 & 1 & 1 & 1 & 1 & 1 & 0 & 0 & 1 & 0 & 11110.7516 & $\odot .0035$ & 0.002 \\
\hline 71: & 2 & 2 & 0 & 1 & 3 & 4 & 1 & 1 & $\odot$ & 0 & 3 & 3 & 11110.8305 & $\odot .0006$ & 0.002 \\
\hline 72: & 2 & 2 & $\odot$ & 1 & 3 & 4 & 1 & 1 & $\odot$ & 0 & 3 & 4 & 11112.0135 & $\odot .0 \odot 1 \odot$ & $\odot .002$ \\
\hline 73: & 2 & 2 & $\odot$ & 1 & 1 & 1 & 1 & 1 & $\odot$ & 0 & 1 & 1 & 11112.2427 & $-\odot . \odot \odot 23$ & $\odot . \odot \odot 2$ \\
\hline 74: & 2 & 2 & 0 & 1 & 2 & 1 & 1 & 1 & $\odot$ & 0 & 2 & 1 & 11113.6334 & $\odot .0026$ & 0.002 \\
\hline 75: & 2 & 2 & 0 & 1 & 3 & 2 & 1 & 1 & 0 & 0 & 1 & 2 & 11113.9338 & $-0.0 \odot 29$ & $\odot .002$ \\
\hline 76: & 2 & 2 & 0 & 1 & 2 & 3 & 1 & 1 & $\odot$ & 0 & 2 & 2 & 11114.3044 & $\odot .0004$ & 0.002 \\
\hline 77: & 2 & 2 & $\odot$ & 1 & 2 & 3 & 1 & 1 & $\odot$ & 0 & 2 & 3 & 11114.4713 & $\odot . \odot \odot \odot 7$ & $\odot .002$ \\
\hline 78: & 2 & 2 & $\odot$ & 1 & 2 & 4 & 1 & 1 & $\odot$ & 0 & 2 & 3 & 11114.5273 & $\odot . \odot \odot 17$ & $\odot .0 \odot 2$ \\
\hline 79: & 2 & 2 & $\odot$ & 1 & 1 & 3 & 1 & 1 & $\odot$ & 0 & 1 & 2 & 11114.8934 & $\odot .0014$ & $\odot .0 \odot 2$ \\
\hline 80: & 2 & 2 & $\odot$ & 1 & 3 & 2 & 1 & 1 & $\odot$ & 0 & 1 & 1 & 11114.9175 & $-\odot .0006$ & $\odot .0 \odot 2$ \\
\hline
\end{tabular}




\begin{tabular}{|c|c|c|c|c|c|c|c|c|c|c|c|}
\hline 1: & 2 & 2 & $\odot$ & 1 & 2 & 1 & 1 & 1 & $\odot$ & $\odot$ & $\odot$ \\
\hline 82: & 2 & 2 & $\odot$ & 1 & 3 & 2 & 1 & 1 & $\odot$ & $\odot$ & 3 \\
\hline 83: & 2 & 2 & $\odot$ & 1 & 1 & 3 & 1 & 1 & $\odot$ & 0 & 3 \\
\hline 84: & 2 & 2 & $\odot$ & 1 & 3 & 5 & 1 & 1 & $\odot$ & $\odot$ & 3 \\
\hline 85: & 2 & 2 & $\odot$ & 1 & 2 & 2 & 1 & 1 & $\odot$ & 0 & 2 \\
\hline 86: & 2 & 2 & $\odot$ & 1 & 3 & 1 & 1 & 1 & $\odot$ & $\odot$ & 1 \\
\hline 87: & 2 & 2 & $\odot$ & 1 & 2 & 2 & 1 & 1 & $\odot$ & $\odot$ & 0 \\
\hline 88: & 2 & 2 & $\odot$ & 1 & 2 & 2 & 1 & 1 & $\odot$ & $\odot$ & 3 \\
\hline 89: & 2 & 2 & $\odot$ & 1 & 1 & 2 & 1 & 1 & $\odot$ & $\odot$ & 1 \\
\hline 90 : & 2 & 2 & $\odot$ & 1 & 1 & 2 & 1 & 1 & $\odot$ & $\odot$ & 0 \\
\hline 91: & 2 & 2 & $\odot$ & 1 & 1 & 2 & 1 & 1 & $\odot$ & $\odot$ & 3 \\
\hline 92: & 2 & 2 & 1 & $\odot$ & 3 & 2 & 1 & 1 & 1 & 1 & 1 \\
\hline 93: & 2 & 2 & 1 & $\odot$ & 3 & 3 & 1 & 1 & 1 & 1 & 1 \\
\hline 94: & 2 & 2 & 1 & $\odot$ & 1 & 2 & 1 & 1 & 1 & 1 & 3 \\
\hline 95: & 2 & 2 & 1 & $\odot$ & $\odot$ & 2 & 1 & 1 & 1 & 1 & 0 \\
\hline 96: & 2 & 2 & 1 & $\odot$ & 1 & 2 & 1 & 1 & 1 & 1 & $\odot$ \\
\hline 97: & 2 & 2 & 1 & $\odot$ & 3 & 1 & 1 & 1 & 1 & 1 & 3 \\
\hline 98: & 2 & 2 & 1 & 1 & 1 & 1 & 1 & 1 & 1 & $\odot$ & 1 \\
\hline 99: & 2 & 2 & 1 & 1 & 3 & 2 & 1 & 1 & 1 & 0 & 3 \\
\hline 100: & 2 & 2 & 1 & $\odot$ & 2 & 3 & 1 & 1 & 1 & 1 & 2 \\
\hline 101: & 2 & 2 & 1 & $\odot$ & 2 & 4 & 1 & 1 & 1 & 1 & 2 \\
\hline 102: & 2 & 2 & 1 & $\odot$ & 3 & 5 & 1 & 1 & 1 & 1 & 3 \\
\hline 103: & 2 & 2 & 1 & $\odot$ & 3 & 3 & 1 & 1 & 1 & 1 & 3 \\
\hline 104: & 2 & 2 & 1 & 1 & 2 & 1 & 1 & 1 & 1 & $\odot$ & $\odot$ \\
\hline 105: & 2 & 2 & 1 & $\odot$ & 0 & 2 & 1 & 1 & 1 & 1 & 1 \\
\hline 106: & 2 & 2 & 1 & 1 & 3 & 4 & 1 & 1 & 1 & $\odot$ & 3 \\
\hline 107: & 2 & 2 & 1 & 1 & 1 & 3 & 1 & 1 & 1 & $\odot$ & 3 \\
\hline 108: & 2 & 2 & 1 & $\odot$ & 2 & 2 & 1 & 1 & 1 & 1 & 2 \\
\hline 109: & 2 & 2 & 1 & $\odot$ & 1 & 2 & 1 & 1 & 1 & 1 & 1 \\
\hline 110: & 2 & 2 & 1 & $\odot$ & 3 & 4 & 1 & 1 & 1 & 1 & 3 \\
\hline 111: & 2 & 2 & 1 & 1 & 2 & 2 & 1 & 1 & 1 & $\odot$ & 2 \\
\hline 112: & 2 & 2 & 1 & 1 & 3 & 2 & 1 & 1 & 1 & $\odot$ & 1 \\
\hline 113: & 2 & 2 & 1 & $\odot$ & 3 & 2 & 1 & 1 & 1 & 1 & 1 \\
\hline 114: & 2 & 2 & 1 & 0 & 1 & 1 & 1 & 1 & 1 & 1 & 1 \\
\hline 115: & 2 & 2 & 1 & 0 & 0 & 2 & 1 & 1 & 1 & 1 & 2 \\
\hline 116: & 2 & 2 & 1 & $\odot$ & 1 & 3 & 1 & 1 & 1 & 1 & 1 \\
\hline 117: & 2 & 2 & 1 & 1 & 3 & 3 & 1 & 1 & 1 & 0 & 1 \\
\hline 118: & 2 & 2 & 1 & 1 & 1 & 2 & 1 & 1 & 1 & $\odot$ & 3 \\
\hline 119: & 2 & 2 & 1 & 1 & 0 & 2 & 1 & 1 & 1 & $\odot$ & 0 \\
\hline 120: & 2 & 2 & 1 & $\odot$ & 3 & 2 & 1 & 1 & 1 & 1 & 3 \\
\hline 121: & 2 & 2 & 1 & 1 & 1 & 2 & 1 & 1 & 1 & $\odot$ & $\odot$ \\
\hline 122: & 2 & 2 & 1 & 1 & 3 & 1 & 1 & 1 & 1 & $\odot$ & 3 \\
\hline 123: & 2 & 2 & 1 & 1 & 2 & 3 & 1 & 1 & 1 & $\odot$ & 2 \\
\hline 124: & 2 & 2 & 1 & 1 & 2 & 3 & 1 & 1 & 1 & $\odot$ & 2 \\
\hline 125: & 2 & 2 & 1 & 1 & 2 & 4 & 1 & 1 & 1 & 0 & 2 \\
\hline 126: & 2 & 2 & 1 & $\odot$ & 1 & 3 & 1 & 1 & 1 & 1 & 3 \\
\hline 127: & 2 & 2 & 1 & 1 & 3 & 5 & 1 & 1 & 1 & $\odot$ & 3 \\
\hline 128: & 2 & 2 & 1 & 1 & 3 & 3 & 1 & 1 & 1 & $\odot$ & 3 \\
\hline 129: & 2 & 2 & 1 & 1 & 0 & 2 & 1 & 1 & 1 & $\odot$ & 1 \\
\hline 130: & 2 & 2 & 1 & 1 & 2 & 2 & 1 & 1 & 1 & $\odot$ & 2 \\
\hline 131: & 2 & 2 & 1 & 1 & 1 & 2 & 1 & 1 & 1 & $\odot$ & 1 \\
\hline 132: & 2 & 2 & 1 & 1 & 3 & 4 & 1 & 1 & 1 & 0 & 3 \\
\hline 133: & 2 & 2 & 1 & 1 & 3 & 2 & 1 & 1 & 1 & $\odot$ & 1 \\
\hline 134: & 2 & 2 & 1 & 1 & 0 & 2 & 1 & 1 & 1 & $\odot$ & 2 \\
\hline 135: & 2 & 2 & 1 & 1 & 1 & 3 & 1 & 1 & 1 & $\odot$ & 1 \\
\hline 136: & 2 & 2 & 1 & 1 & 3 & 2 & 1 & 1 & 1 & $\odot$ & 3 \\
\hline 137: & 2 & 2 & 1 & 1 & 2 & 1 & 1 & 1 & 1 & $\odot$ & 2 \\
\hline 138: & 2 & 2 & 1 & 1 & 1 & 3 & 1 & 1 & 1 & $\odot$ & 3 \\
\hline 139: & 3 & 2 & 1 & $\odot$ & 3 & 5 & 2 & 1 & 1 & 1 & 3 \\
\hline 140: & 3 & 2 & 1 & $\odot$ & 1 & 2 & 2 & 1 & 1 & 1 & 1 \\
\hline 141: & 3 & 2 & 1 & $\odot$ & 2 & 3 & 2 & 1 & 1 & 1 & 2 \\
\hline 142: & 3 & 2 & 1 & $\odot$ & 3 & 4 & 2 & 1 & 1 & 1 & 3 \\
\hline 143: & 3 & 2 & 1 & $\odot$ & 2 & 5 & 2 & 1 & 1 & 1 & 2 \\
\hline 144: & 3 & 2 & 1 & $\odot$ & 2 & 4 & 2 & 1 & 1 & 1 & 2 \\
\hline 145: & 3 & 2 & 1 & $\odot$ & 3 & 6 & 2 & 1 & 1 & 1 & 3 \\
\hline 146: & 3 & 2 & 1 & 0 & 3 & 4 & 2 & 1 & 1 & 1 & 3 \\
\hline 147: & 3 & 2 & 1 & 0 & 1 & 4 & 2 & 1 & 1 & 1 & 1 \\
\hline 148: & 3 & 2 & 1 & 0 & $\odot$ & 3 & 2 & 1 & 1 & 1 & $\odot$ \\
\hline 149: & 3 & 2 & 1 & 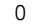 & 1 & 3 & 2 & 1 & 1 & 1 & 1 \\
\hline 150: & 3 & 2 & 1 & $\odot$ & 3 & 3 & 2 & 1 & 1 & 1 & 3 \\
\hline 151: & 3 & 2 & 1 & 1 & 3 & 5 & 2 & 1 & 1 & 0 & 3 \\
\hline 152: & 3 & 2 & 1 & 1 & 1 & 2 & 2 & 1 & 1 & $\odot$ & 1 \\
\hline 153: & 3 & 2 & 1 & 1 & 2 & 3 & 2 & 1 & 1 & $\odot$ & 2 \\
\hline 154: & 3 & 2 & 1 & 1 & 3 & 4 & 2 & 1 & 1 & $\odot$ & 3 \\
\hline 155: & 3 & 2 & 1 & 1 & 2 & 5 & 2 & 1 & 1 & $\odot$ & 2 \\
\hline 156: & 3 & 2 & 1 & 1 & 2 & 4 & 2 & 1 & 1 & $\odot$ & 2 \\
\hline 157: & 3 & 2 & 1 & 1 & 3 & 6 & 2 & 1 & 1 & $\odot$ & 3 \\
\hline 158: & 3 & 2 & 1 & 1 & 3 & 4 & 2 & 1 & 1 & $\odot$ & 3 \\
\hline 159: & 3 & 2 & 1 & 1 & 1 & 4 & 2 & 1 & 1 & $\odot$ & 1 \\
\hline 160: & 3 & 2 & 1 & 1 & $\odot$ & 3 & 2 & 1 & 1 & 0 & 0 \\
\hline 161: & 3 & 2 & 1 & 1 & 1 & 3 & 2 & 1 & 1 & $\odot$ & 1 \\
\hline 162: & 3 & 2 & 1 & 1 & 3 & 3 & 2 & 1 & 1 & $\odot$ & 3 \\
\hline 163: & 3 & 2 & 1 & 1 & 3 & 5 & 2 & 1 & 1 & $\odot$ & 3 \\
\hline 164: & 3 & 2 & 2 & $\odot$ & 3 & 6 & 2 & 1 & 2 & 1 & 3 \\
\hline 165: & 3 & 2 & 2 & $\odot$ & 1 & 4 & 2 & 1 & 2 & 1 & 1 \\
\hline 166: & 3 & 2 & 2 & $\odot$ & 2 & 4 & 2 & 1 & 2 & 1 & 2 \\
\hline 167: & 3 & 2 & 2 & $\odot$ & 2 & 5 & 2 & 1 & 2 & 1 & 2 \\
\hline
\end{tabular}




\begin{tabular}{|c|c|c|c|c|c|c|c|c|c|c|c|}
\hline 168: & 3 & 2 & 2 & $\odot$ & 3 & 5 & 2 & 1 & 2 & 1 & 3 \\
\hline 169: & 3 & 2 & 2 & $\odot$ & $\odot$ & 3 & 2 & 1 & 2 & 1 & 2 \\
\hline $170 /$ & 3 & 2 & 2 & $\odot$ & 1 & 4 & 2 & 1 & 2 & 1 & 3 \\
\hline 171: & 3 & 2 & 2 & 1 & 3 & 6 & 2 & 1 & 2 & $\odot$ & 3 \\
\hline 172: & 3 & 2 & 2 & 1 & 1 & 4 & 2 & 1 & 2 & $\odot$ & 1 \\
\hline 73: & 3 & 2 & 2 & 1 & 1 & 3 & 2 & 1 & 2 & $\odot$ & 3 \\
\hline $74:$ & 3 & 2 & 2 & 1 & 2 & 4 & 2 & 1 & 2 & $\odot$ & 2 \\
\hline 75: & 3 & 2 & 2 & 1 & 2 & 5 & 2 & 1 & 2 & $\odot$ & 2 \\
\hline 76 : & 3 & 2 & 2 & 1 & 3 & 5 & 2 & 1 & 2 & $\odot$ & 3 \\
\hline 77: & 3 & 2 & 2 & 1 & $\odot$ & 3 & 2 & 1 & 2 & $\odot$ & 2 \\
\hline 78: & 3 & 2 & 2 & 1 & 1 & 4 & 2 & 1 & 2 & $\odot$ & 3 \\
\hline 79: & 3 & 1 & 2 & $\odot$ & 3 & 1 & 2 & $\odot$ & 2 & 1 & 3 \\
\hline 80 : & 3 & 1 & 2 & 0 & 3 & 2 & 2 & 0 & 2 & 1 & 3 \\
\hline 81: & 3 & 1 & 2 & 0 & 1 & 3 & 2 & 0 & 2 & 1 & 1 \\
\hline 82: & 3 & 1 & 2 & 0 & $\odot$ & 3 & 2 & $\odot$ & 2 & 1 & 2 \\
\hline 83: & 3 & 1 & 2 & $\odot$ & 3 & 6 & 2 & 0 & 2 & 1 & 3 \\
\hline 184: & 3 & 1 & 2 & $\odot$ & 3 & 2 & 2 & 0 & 2 & 1 & 1 \\
\hline 185: & 3 & 1 & 2 & $\odot$ & 1 & 4 & 2 & $\odot$ & 2 & 1 & 1 \\
\hline 186: & 3 & 1 & 2 & $\odot$ & 3 & 2 & 2 & 0 & 2 & 1 & 3 \\
\hline 187: & 3 & 1 & 2 & $\odot$ & 2 & 5 & 2 & 0 & 2 & 1 & 2 \\
\hline 188: & 3 & 1 & 2 & $\odot$ & 2 & 4 & 2 & 0 & 2 & 1 & 2 \\
\hline 89: & 3 & 1 & 2 & $\odot$ & 3 & 3 & 2 & 0 & 2 & 1 & 3 \\
\hline $90:$ & 3 & 1 & 2 & $\odot$ & 3 & 5 & 2 & 0 & 2 & 1 & 3 \\
\hline 191: & 3 & 1 & 2 & $\odot$ & 1 & 2 & 2 & $\odot$ & 2 & 1 & 1 \\
\hline L92: & 3 & 1 & 2 & $\odot$ & 3 & 3 & 2 & 0 & 2 & 1 & 3 \\
\hline 93: & 3 & 1 & 2 & 1 & 3 & $\odot$ & 2 & 0 & 2 & $\odot$ & 3 \\
\hline 94: & 3 & 1 & 2 & $\odot$ & 2 & 3 & 2 & $\odot$ & 2 & 1 & $\odot$ \\
\hline 95: & 3 & 1 & 2 & $\odot$ & 3 & 4 & 2 & 0 & 2 & 1 & 3 \\
\hline 196: & 3 & 1 & 2 & $\odot$ & 3 & 4 & 2 & $\odot$ & 2 & 1 & 3 \\
\hline $197 /$ & 3 & 1 & 2 & 0 & 3 & 5 & 2 & $\odot$ & 2 & 1 & 3 \\
\hline 198: & 3 & 1 & 2 & 1 & 3 & 1 & 2 & $\odot$ & 2 & $\odot$ & 3 \\
\hline 199: & 3 & 1 & 2 & 1 & 3 & 2 & 2 & 0 & 2 & $\odot$ & 3 \\
\hline 200: & 3 & 1 & 2 & 1 & 1 & 3 & 2 & $\odot$ & 2 & $\odot$ & 1 \\
\hline 201: & 3 & 1 & 2 & 1 & $\odot$ & 3 & 2 & $\odot$ & 2 & 0 & 2 \\
\hline 202: & 3 & 1 & 2 & 1 & 3 & 6 & 2 & 0 & 2 & $\odot$ & 3 \\
\hline 203: & 3 & 1 & 2 & 1 & 1 & 4 & 2 & 0 & 2 & 0 & 1 \\
\hline 204: & 3 & 1 & 2 & 1 & 3 & 2 & 2 & $\odot$ & 2 & $\odot$ & 3 \\
\hline 205: & 3 & 1 & 2 & 1 & 2 & 5 & 2 & 0 & 2 & $\odot$ & 2 \\
\hline 206: & 3 & 1 & 2 & 1 & 2 & 4 & 2 & $\odot$ & 2 & 0 & 2 \\
\hline 207: & 3 & 1 & 2 & 1 & 3 & 3 & 2 & 0 & 2 & $\odot$ & 3 \\
\hline 208: & 3 & 1 & 2 & 1 & 3 & 5 & 2 & 0 & 2 & 0 & 3 \\
\hline 209: & 3 & 1 & 2 & 1 & 1 & 2 & 2 & 0 & 2 & $\odot$ & 1 \\
\hline 210: & 3 & 1 & 2 & 1 & 3 & 3 & 2 & $\odot$ & 2 & 0 & 3 \\
\hline 211: & 3 & 1 & 2 & 1 & 2 & 3 & 2 & $\odot$ & 2 & $\odot$ & $\odot$ \\
\hline 212: & 3 & 1 & 2 & 1 & 3 & 4 & 2 & 0 & 2 & $\odot$ & 3 \\
\hline 213: & 3 & 1 & 2 & 1 & 3 & 4 & 2 & $\odot$ & 2 & $\odot$ & 3 \\
\hline 214: & 3 & 1 & 2 & 1 & 3 & 5 & 2 & $\odot$ & 2 & $\odot$ & 3 \\
\hline 215: & 3 & 3 & $\odot$ & $\odot$ & 3 & 5 & 2 & 2 & $\odot$ & 1 & 3 \\
\hline 216: & 3 & 3 & $\odot$ & $\odot$ & 3 & 4 & 2 & 2 & $\odot$ & 1 & 3 \\
\hline 217: & 3 & 3 & $\odot$ & $\odot$ & 3 & 4 & 2 & 2 & $\odot$ & 1 & 3 \\
\hline 218: & 3 & 3 & $\odot$ & $\odot$ & 2 & 3 & 2 & 2 & $\odot$ & 1 & $\odot$ \\
\hline 219: & 3 & 3 & $\odot$ & $\odot$ & 3 & 3 & 2 & 2 & $\odot$ & 1 & 3 \\
\hline 220: & 3 & 3 & $\odot$ & $\odot$ & 1 & 2 & 2 & 2 & $\odot$ & 1 & 1 \\
\hline 221: & 3 & 3 & $\odot$ & $\odot$ & 3 & 5 & 2 & 2 & $\odot$ & 1 & 3 \\
\hline 222: & 3 & 3 & $\odot$ & $\odot$ & 2 & 4 & 2 & 2 & $\odot$ & 1 & 2 \\
\hline 223: & 3 & 3 & $\odot$ & $\odot$ & 2 & 5 & 2 & 2 & $\odot$ & 1 & 2 \\
\hline 224: & 3 & 3 & $\odot$ & $\odot$ & 3 & 3 & 2 & 2 & $\odot$ & 1 & 3 \\
\hline 225: & 3 & 3 & $\odot$ & $\odot$ & 1 & 4 & 2 & 2 & $\odot$ & 1 & 1 \\
\hline 226: & 3 & 3 & $\odot$ & $\odot$ & 3 & 6 & 2 & 2 & $\odot$ & 1 & 3 \\
\hline 227: & 3 & 3 & $\odot$ & $\odot$ & $\odot$ & 3 & 2 & 2 & $\odot$ & 1 & 2 \\
\hline 228: & 3 & 3 & $\odot$ & $\odot$ & 1 & 3 & 2 & 2 & $\odot$ & 1 & 1 \\
\hline 229: & 3 & 3 & $\odot$ & $\odot$ & 3 & 1 & 2 & 2 & $\odot$ & 1 & 3 \\
\hline 230: & 3 & 3 & $\odot$ & $\odot$ & 3 & 2 & 2 & 2 & $\odot$ & 1 & 3 \\
\hline 231: & 3 & 3 & $\odot$ & 1 & 3 & 4 & 2 & 2 & $\odot$ & 0 & 3 \\
\hline 232: & 3 & 3 & $\odot$ & 1 & 3 & 4 & 2 & 2 & $\odot$ & $\odot$ & 3 \\
\hline 233: & 3 & 3 & $\odot$ & 1 & 2 & 3 & 2 & 2 & $\odot$ & 0 & 0 \\
\hline 234: & 3 & 3 & $\odot$ & 1 & 3 & 3 & 2 & 2 & $\odot$ & $\odot$ & 3 \\
\hline 235: & 3 & 3 & $\odot$ & 1 & 1 & 2 & 2 & 2 & 0 & 0 & 1 \\
\hline 236: & 3 & 3 & $\odot$ & 1 & 3 & 5 & 2 & 2 & 0 & 0 & 3 \\
\hline 237: & 3 & 3 & $\odot$ & 1 & 2 & 4 & 2 & 2 & $\odot$ & $\odot$ & 2 \\
\hline 238: & 3 & 3 & $\odot$ & 1 & 2 & 5 & 2 & 2 & 0 & 0 & 2 \\
\hline 239: & 3 & 3 & $\odot$ & 1 & 3 & 3 & 2 & 2 & 0 & $\odot$ & 3 \\
\hline 240: & 3 & 3 & $\odot$ & 1 & 1 & 4 & 2 & 2 & $\odot$ & $\odot$ & 1 \\
\hline 241: & 3 & 3 & $\odot$ & 1 & 3 & 6 & 2 & 2 & $\odot$ & $\odot$ & 3 \\
\hline 242: & 3 & 3 & $\odot$ & 1 & $\odot$ & 3 & 2 & 2 & 0 & $\odot$ & 2 \\
\hline 243: & 3 & 3 & $\odot$ & 1 & 1 & 3 & 2 & 2 & 0 & 0 & 1 \\
\hline 244: & 3 & 3 & 1 & 0 & 3 & 4 & 2 & 2 & 1 & 1 & 3 \\
\hline 245: & 3 & 3 & 1 & 0 & 1 & 4 & 2 & 2 & 1 & 1 & 1 \\
\hline 246: & 3 & 3 & 1 & 0 & 2 & 3 & 2 & 2 & 1 & 1 & 2 \\
\hline 247: & 3 & 3 & 1 & 0 & 2 & 4 & 2 & 2 & 1 & 1 & 2 \\
\hline 248: & 3 & 3 & 1 & 0 & 2 & 5 & 2 & 2 & 1 & 1 & 2 \\
\hline 249: & 3 & 3 & 1 & $\odot$ & 1 & 2 & 2 & 2 & 1 & 1 & 1 \\
\hline 250: & 3 & 3 & 1 & 0 & 1 & 2 & ? & 2 & 1 & 1 & 1 \\
\hline 251: & 3 & 3 & 1 & $\odot$ & $\odot$ & 3 & 2 & 2 & 1 & 1 & $\odot$ \\
\hline 252: & 3 & 3 & 1 & 0 & 3 & 5 & 2 & 2 & 1 & 1 & 3 \\
\hline 253: & 3 & 3 & 1 & 0 & 3 & 6 & 2 & 2 & 1 & 1 & 3 \\
\hline 254 & 3 & 3 & 1 & 1 & 3 & 3 & 2 & 2 & 1 & 0 & 3 \\
\hline
\end{tabular}


$\begin{array}{llllllllllllllll}255: & 3 & 3 & 1 & 1 & 3 & 4 & 2 & 2 & 1 & 0 & 3 & 3 & 17380.5333 & -0.0004 & 0.002\end{array}$

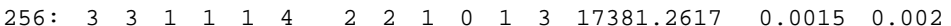

257: $\begin{array}{llllllllllllllll}3 & 3 & 1 & 1 & 2 & 3 & 2 & 2 & 1 & 0 & 2 & 2 & 17381.5606 & -0.0030 & 0.002\end{array}$

258: $3 \begin{array}{lllllllllllllll}3 & 3 & 1 & 1 & 2 & 4 & 2 & 2 & 1 & 0 & 2 & 3 & 17381.9279 & -0.0019 & 0.002\end{array}$

259: $3 \begin{array}{lllllllllllllll}3 & 3 & 1 & 1 & 1 & 3 & 2 & 2 & 1 & 0 & 1 & 2 & 17382.1875 & -0.0046 & 0.002\end{array}$

$\begin{array}{llllllllllllllll}260: & 3 & 3 & 1 & 1 & 2 & 5 & 2 & 2 & 1 & 0 & 2 & 4 & 17382.1987 & 0.0002 & 0.002\end{array}$

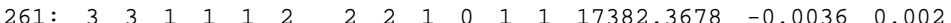

$\begin{array}{llllllllllllllll}262: & 3 & 3 & 1 & 1 & 0 & 3 & 2 & 2 & 1 & 0 & 0 & 2 & 17382.7368 & -0.0024 & 0.002\end{array}$

$\begin{array}{llllllllllllllll}263: & 3 & 3 & 1 & 1 & 3 & 5 & 2 & 2 & 1 & 0 & 3 & 4 & 17383.1369 & -0.0010 & 0.002\end{array}$

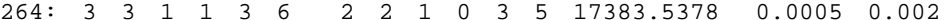

$\begin{array}{llllllllllllllll}265 / & 3 & 3 & 1 & 1 & 3 & 3 & 2 & 2 & 1 & 0 & 3 & 3 & 17387.1614 & -0.0067 & 0.002\end{array}$ 